\title{
Bacterial inhibition of CD8+ T-cells mediated cell death promotes neuroinvasion and within-host persistence
}

\author{
Marc Lecuit ( $\square$ marc.lecuit@pasteur.fr) \\ Institut Pasteur https://orcid.org/0000-0002-4491-1063 \\ Claire Maudet \\ Institut Pasteur https://orcid.org/0000-0001-8856-0819 \\ Marouane Kheloufi \\ Institut Pasteur \\ Sylvain Levallois \\ Institut Pasteur https://orcid.org/0000-0002-5805-1092 \\ Julien Gaillard \\ Institut Pasteur \\ Lei Huang \\ Institut Pasteur \\ Charlotte Gaultier \\ Institut Pasteur \\ Yu-Huan Tsai \\ Institut Pasteur \\ Olivier Disson \\ Institut Pasteur
}

\section{Biological Sciences - Article}

Keywords: Central nervous system infections, Listeria monocytogenes, neuroinvasion

Posted Date: January 14th, 2021

DOl: https://doi.org/10.21203/rs.3.rs-112606/v1

License: (c) (i) This work is licensed under a Creative Commons Attribution 4.0 International License. Read Full License

Version of Record: A version of this preprint was published at Nature on March 16th, 2022. See the published version at https://doi.org/10.1038/s41586-022-04505-7. 

5

6

7

8

9

\section{Bacterial inhibition of $\mathrm{CD8}^{+} \mathrm{T}$-cells mediated cell death promotes neuroinvasion and} within-host persistence

Claire Maudet $^{1,2, *}$, Marouane Kheloufi ${ }^{1,2, *}$, Sylvain Levallois ${ }^{1,2,3}$, Julien Gaillard ${ }^{1,2,3}$, Lei Huang ${ }^{1,2,3}$, Charlotte Gaultier ${ }^{1,2,3}$, Yu-Huan Tsai ${ }^{1,2, \$}$, Olivier Disson ${ }^{1,2}$, Marc Lecuit ${ }^{1,2,3,4,5, \dagger}$

${ }^{1}$ Institut Pasteur, Biology of Infection Unit, Inserm U1117, 75015, Paris, France

${ }^{2}$ Inserm U1117, 75015, Paris, France

${ }^{3}$ Université de Paris, 75006, Paris, France

${ }^{4}$ National Reference Centre and WHO Collaborating Centre Listeria, Institut Pasteur, 75015, Paris, France

${ }^{5}$ Necker-Enfants Malades University Hospital, Division of Infectious Diseases and Tropical Medicine, APHP, Institut Imagine, 75006, Paris, France

* These authors share first authorship

${ }^{\dagger}$ Correspondence to: marc.lecuit@pasteur.fr

* Current address: Institute of Microbiology and Immunology, National Yang-Ming University, Taipei, Taiwan 
46

\section{Abstract}

Central nervous system infections are amongst the most severe ${ }^{1,2}$, yet the mechanisms by which pathogens access the brain remain poorly understood. The model microorganism Listeria monocytogenes $(\mathrm{Lm})$ is a major foodborne pathogen that causes neurolisteriosis, one of the deadliest central nervous system infections ${ }^{3,4}$. While immunosuppression is a well-established host risk factor for neurolisteriosis ${ }^{3,5}$, little is known regarding the bacterial factors underlying $L m$ neuroinvasion. We have developed a clinically-relevant experimental model of neurolisteriosis, using hypervirulent neuroinvasive strains ${ }^{6}$ inoculated in a humanized mouse model of infection ${ }^{7}$, and we show that the bacterial protein InlB protects infected monocytes from $\mathrm{CD}^{+}$T-cells Fas-mediated cell death, in a c-Met/PI3-kinase/FLIP-dependent manner. This blockade of anti- $L m$ specific cellular immune response lengthens infected monocytes lifespan, favoring $L m$ transfer from infected monocytes to the brain. The intracellular niche created by InlB-mediated cell-autonomous immunosuppression also promotes $\mathrm{Lm}$ fecal shedding, accounting for its selection as a $L m$ core virulence gene. Here, we have uncovered an unanticipated specific mechanism by which a bacterial pathogen confers to the cells it infects an increased lifespan by rendering them resistant to cell-mediated immunity. This promotes $\mathrm{Lm}$ within-host persistence and dissemination to the central nervous system, and transmission.

One Sentence Summary: Listeria blocks $\mathrm{CD} 8^{+} \mathrm{T}$-cells killing and promotes neuroinvasion 
Bacterial infections of the central nervous system $(\mathrm{CNS})$ are often fatal ${ }^{1,2}$, yet little is known regarding the molecular mechanisms underlying microbial neuroinvasion. Listeria monocytogenes $(\mathrm{Lm})$ is a foodborne pathogen that causes neurolisteriosis, one of the deadliest CNS infections ${ }^{3,4}$. Consistent with its key role in immunity against $L m$, T-cell based immunosuppression is a major host risk factor for neurolisteriosis $\mathrm{s}^{3,5}$. In contrast, the bacterial factors promoting $\mathrm{Lm}$ neuroinvasion and their mechanisms of action are poorly understood. Earlier studies have pointed towards the involvement of monocytes in transferring $\mathrm{Lm}$ from the blood to the brain ${ }^{8,9}$. However, these investigations were performed with reference laboratory $L m$ strains, which are actually poorly neuroinvasive ${ }^{6}$, and require very high bacterial inocula to induce some degree of CNS infection in experimental animal models. This is consistent with the observation that these strains belong to clonal complexes very rarely responsible for human neurolisteriosis ${ }^{3,6}$. In contrast, clinically-associated clonal complexes are hypervirulent and more neuroinvasive ${ }^{6}$. In order to investigate the mechanisms underlying $\mathrm{Lm}$ neuroinvasion, we developed a clinically-relevant experimental model of neurolisteriosis based on the inoculation of hypervirulent neuroinvasive $L m$ strains $^{6}$ in a humanized mouse model ${ }^{7}$. 


\section{Infected inflammatory monocytes mediate $L m$ neuroinvasion}

We inoculated orally $\mathrm{Lm}$ in humanized KIE16P mice ${ }^{7}$. In contrast to the reference laboratory strain EGDe that belongs to clonal complex (CC) $9^{10,11}$, clinical isolates belonging to the hypervirulent clonal complexes $\mathrm{CC} 1,4$ and 6 systematically induce high-level neuroinvasion, as previously reported ${ }^{6}$, starting at 3 days post-inoculation (dpi) (Fig. 1a). At 5 dpi, the bacterial brain load is the same with or without administration of gentamicin (Fig. 1b), an antibiotic that kills extracellular but not intracellular Lm (Extended Data Fig. 1a, b), indicating that intracellular bacteria are involved in neuroinvasion. Consistent with the occurrence of neuroinvasion in the presence of gentamicin, $L m$ is detected intracellularly in the blood and

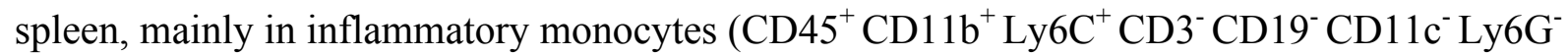
) (Fig. 1c, d and Extended Data Fig. 1c-e), suggesting that monocytes are involved in $\mathrm{Lm}$ neuroinvasion. This was assessed by infecting $\mathrm{Ccr} 2^{-/-}$mice, in which monocytes are retained in the bone marrow and are therefore less abundant in the blood and spleen ${ }^{12}$ (Extended Data Fig. 1f). Indeed, from day 1 to 3 post-inoculation, more bacteria are gradually recovered in the brain of WT mice as compared to $C c r 2^{-/-}$mice (Fig. 1e). Moreover, the transfer of infected monocytes from donor-infected mice into gentamicin-treated uninfected recipient mice is sufficient to induce neuroinvasion, and $\mathrm{Lm}$ can be recovered from the brain of recipient mice as early as day 2 post-transfer (Fig. 1f). In contrast, the transfer of infected monocytes from mice expressing the diphteria toxin (DT) receptor in myeloid cells $\left(\right.$ LysM-CreER $\left.{ }^{\mathrm{T} 2} \times \mathrm{iDTR}\right)$ into recipient mice treated with DT leads to liver and spleen infection but no brain infection, even as late as 4 days post-transfer (Extended Data Fig. 1g, h). Together, these results indicate that infected monocytes are necessary and sufficient to induce neuroinvasion.

Infected monocytes were observed adhering to the endothelium of blood vessels in brain sections of infected mice (Fig. 1g and Extended Data Fig. 2a-c). In these adhering monocytes, Lm polymerizing actin was observed (Extended Data Fig. $2 \mathrm{~d}$ and movie S1), significantly more 
than in spleen monocytes (Extended Data Fig. 2e, f), and occasionally adjacent to infected endothelial cells (Fig. 1h and movie S1). Moreover, the transfer of monocytes infected with Lm $\triangle a c t A$ isogenic mutant, unable to polymerize actin and mediate cell-to-cell spread ${ }^{13,14}$, fails to induce neuroinvasion (Fig. 1f). Together, these results demonstrate that $L m$ accesses the brain parenchyma by cell-to-cell spread from adhering bloodborne infected inflammatory monocytes (Fig. 1i). These results are in line with previous reports ${ }^{8,9}$ obtained using poorly-neuroinvasive $L m$ strains, suggesting that neuroinvasive $L m$ strains invade the CNS in a similar manner, albeit to a far greater efficiency (up to 3 orders of magnitude) (Fig 1a).

\section{InIB increases the number of infected inflammatory monocytes and mediates}

\section{neuroinvasion}

Having identified infected monocytes as critically involved in the onset of neurolisteriosis, we aimed to identify the bacterial factors responsible for $L m$ neuroinvasiveness. Given the wellestablished role of InlA and InlB in $L m$ crossing of host barriers ${ }^{7,15}$, we investigated their respective role in neuroinvasion. To bypass the well-established role of InlA in the crossing of the intestinal barrier, we inoculated KIE16P mice via the iv route. While InlA plays no role in neuroinvasion (Fig. 2a, Extended Data Fig. 3a-d), InlB plays a major role: the $\Delta i n l B$ mutant is significantly less neuroinvasive than its WT parental strain in co-infection experiments (Fig. 2b, Extended Data Fig. 3a, e). Of note, the $\triangle i n l A B$ mutant is not less neuroinvasive than the $\triangle i n l B$ mutant, ruling out that InlA would act in a conjugated manner with InlB, as it has been reported for placental invasion (Extended Data Fig. 3f, g) ${ }^{7}$. After oral inoculation, a similar difference in neuroinvasiveness is observed between all WT neuroinvasive strains and their corresponding $\Delta i n l B$ mutant (Fig. 2c, Extended Data Fig. 3h). The involvement of InlB in neuroinvasion is also observed upon separate inoculation with either WT- $L m$ or $L m \Delta i n l B$ (Fig. 2d, e). InlB contribution to neuroinvasion increases over time (Fig. 2f, g and Extended Data 
Fig. 3i, j) and Lm $\Delta$ inlB never reaches the brain infection level of WT-Lm (Fig. 2f, g). These results were confirmed by gene complementation (Extended Data Fig. 3f, g).

This critical role of $i n l B$ in $L m$ neuroinvasion was unexpected, as this gene is part of $L m$ core genome and is therefore present in all $\mathrm{Lm}$ strains, including the poorly neuroinvasive reference strains EGDe and 10403S. Neuroinvasive $L m$ isolates actually strongly upregulate the inlAB operon as compared to EGDe and 10403S, both in vitro in liquid culture and in vivo in infected spleen (Fig 2h, i and Extended Data Fig. 3k-m). To assess the impact of InlB expression level on neuroinvasiveness, we complemented the EGDe $\Delta$ inlB mutant with the $i n l B$ gene sequence from either EGDe or CC4 (primary sequences share 93\% identity, Extended Data Fig. 4a) so that inlB within-host transcription levels are similar to that of endogenous inlB in CC4 (Extended Data Fig. 4b). These complemented strains are as neuroinvasive as WT-CC4, whereas CC4 $\triangle$ inlB is as poorly neuroinvasive as EGDe $\Delta$ inlB (Fig. $2 \mathrm{j}$ and Extended Data Fig. c, d). Consistently, CC4AinlB complemented with the inlB EGDe allele expressed to the level of CC4 in vivo is as neuroinvasive as WT-CC4 (Fig. 2j and Extended Data Fig. 4 c). Altogether, these results establish that InlB overexpression is the critical factor of $L m$ neuroinvasiveness.

We next evaluated the contribution of InlB to the infection of inflammatory monocytes, having shown their essential role in $L m$ neuroinvasion (Fig. 1). From 3 dpi, the blood bacterial load is higher for WT-Lm than $L m \Delta i n l B$ (Fig. 2k, 1). Moreover, InlB significantly increases the number of $L m$-infected inflammatory monocytes in the blood and spleen (Fig. 2m, n and Extended Data Fig. 5a, b). In addition, InlB-mediated neuroinvasion is abrogated in $\mathrm{Ccr}^{-/-}$mice (Fig. $2 \mathrm{o}$ and Extented Data Fig. 5c), indicating that InlB contribution to neuroinvasion implicates infected monocytes. At early time points, when equal numbers of WT and $\Delta i n l B$-bacteria are retrieved from the blood (1-2 dpi, Extended Data Fig. 5d), equivalent numbers of WT- and $\Delta i n l B$-infected adhering monocytes are observed in the brain (Extended Data Fig. 2c), showing that InlB does not alter monocyte ability to adhere to brain blood vessels. Moreover, no impact of InlB on 
bacterial growth is detected upon direct injection of $L m$ into the brain (Extended Data Fig. 5e, f). Altogether, these results indicate that InlB leads to an increased number of circulating infected monocytes, which are themselves required for $L m$ neuroinvasion.

Although InlB has been described as an invasion protein mediating $L m$ internalization into nonphagocytic cells ${ }^{16,17}$, the entry of hypervirulent $L m$ in inflammatory monocytes, which are professional phagocytes, is InlB-independent (Extended Data Fig. 5g-i). This indicates that InlB contribution to neuroinvasion is independent of its capacity to induce internalization.

\section{InIB-mediated neuroinvasion requires a functional adaptive immune system}

T-cell immunosuppression is a well-established risk factor for neurolisteriosis ${ }^{3,5,18-20}$, and hypervirulent $L m$ clones that express the most InlB (Fig. $2 \mathrm{~h}$, i) tend to infect patients that are the least immunosuppressed ${ }^{6}$. We therefore hypothesized that InlB may exhibit immunosuppressive properties. Interestingly, treatment with ciclosporin, a prototypic T-cell immunosuppressant, abrogates InlB-dependent neuroinvasion (Fig. 2c, Fig. 3a, b, and Extended Data Fig. 3h), implying that InlB contribution to $L m$ neuroinvasion requires a functional adaptive immunity. Consistently, no difference in neuroinvasion is observed between WT-Lm and $L m \Delta$ inlB before 3 to $4 \mathrm{dpi}$, at a time when adaptive immune responses are not yet expected to be functional ${ }^{21}$ (Fig. 2f, g and Extended Data Fig. 3i, j). Of note, ciclosporin treatment of EGDe-inoculated mice also leads to a slight increased number of infected inflammatory monocytes (Fig. 3c) and increased neuroinvasion (Fig. 3d, e), in line with an immunomodulatory effect of InlB overexpression in hypervirulent isolates. Strikingly, InlB contribution to neuroinvasion is fully abrogated in Rag2 $2^{-/}$mice (Fig. $3 \mathrm{f}$ and Extended Data Fig. 6a-c), that lack functional lymphoid cells, demonstrating their requirement for InlB to exert its immune-mediated effect. The effect of InlB is also abrogated in $\mathrm{CD} 3 \varepsilon^{-/ /}$, but not in $\mathrm{muMt}^{-/-}$ mice (Fig. 3g and Extended Data Fig. 6d-f), and thus depends on T- but not B-lymphocytes. 
Specifically, $\mathrm{CD} 8^{+}$T-cells depletion fully abrogated the effect of InlB (Fig. 3h and Extended Data Fig. 6g, h). Interestingly, specific anti- $\mathrm{Lm} \mathrm{CD} 8^{+} \mathrm{T}$-cells are induced to the same extent by WT-Lm and $L m \Delta i n l B$ isogenic mutant (Extended Data Fig. 7a-d), and accordingly, mice inoculated with WT-Lm or $L m \Delta i n l B$ display the same level of protective immunity after a second challenge (Extended Data Fig. 7e). Since InlB-mediated neuroinvasion relies on infected monocytes and is detectable in co-infection experiments, we reasoned that InlB may protect specifically infected monocytes from anti- $\mathrm{Lm} \mathrm{T}-\mathrm{CD} 8^{+}$-mediated specific killing. We therefore performed cytotoxic T-lymphocyte (CTL) assays: infected or uninfected inflammatory monocytes and activated $\mathrm{CD}^{+} \mathrm{T}$-cells were retrieved from mice, either uninfected or infected with WT-Lm or $L m \Delta i n l B$, co-incubated and assessed for cell death (Fig. 3i). Strikingly, WT- $L m$-infected monocytes are protected from $\mathrm{CD} 8^{+} \mathrm{T}$-cells-mediated cell death, whereas no difference in cell death is observed in uninfected monocytes (Fig. $3 \mathrm{j}$ and Extended Data Fig. 8a, b). InlB can be either associated to the bacterial surface or released in the surrounding medium ${ }^{17}$. Consistent with a cell-autonomous effect of InlB, the surfaceassociated InlB is required for InlB-mediated neuroinvasion (Extended Data Fig. 8c-f). The fact that infection of monocytes is clonal (Extended Data Fig. 8g) and that InlB contribution to neuroinvasion is detectable in co-infection experiments is also fully consistent with the finding that InlB acts in a cell-autonomous manner.

\section{InIB blocks Fas-mediated cell death, protecting infected cells from killing by $\mathrm{CD8}^{+} \mathrm{T}$ cells}

The cytotoxicity mediated by $\mathrm{CD} 8^{+}$T-cells from either WT- $L m$ and $L m \Delta i n l B$ infected mice is similar (Fig. 3j), confirming that InlB has no impact on immunization (Extended Data Fig. 7). Since $\mathrm{CD}^{+} \mathrm{T}$-cells cytotoxicity relies on the perforin-granzyme and Fas-Fas ligand pathways ${ }^{22}$, we performed CTL assays in Perforin- and Fas-deficient mice (Prfl KO and Fas ${ }^{\text {lpr-cg, }}$ respectively). InlB-mediated protection against monocyte killing by $\mathrm{CD} 8^{+} \mathrm{T}$-cells is fully 
preserved in Prfl KO mice (Extended Data Fig. 8h). In sharp contrast, the inhibitory effect of InlB on infected monocytes cell death is fully abrogated in Fas ${ }^{l p r-c g}$ mice (Extended Data Fig. 8i). This indicates that InlB blocks Fas-mediated killing whereas the perforin pathway is not involved. Consistently, monocytes infected with WT- $L m$, but not $L m \Delta i n l B$, are resistant to FasL-induced apoptosis, while surface expression of Fas is not affected by InlB (Fig. 3k and Extended Data Fig. 8j, k). Accordingly, in mice treated with a pharmacological inhibitor of caspase-8, the downstream effector of $\mathrm{Fas}^{23}$, $L m \Delta i n l B$ becomes as neuroinvasive as WT- $L m$ (Fig. 31, m). In infected mice, the half-life of monocytes infected by WT-Lm is twice longer than that of monocytes infected by Lm $\Delta$ inlB (Extended Data Fig. 81), highlighting that InlB promotes the survival of infected cells. Together, these results demonstrate that InlB blocks $\mathrm{CD} 8^{+}$T-cells Fas-mediated killing of infected monocytes.

\section{InIB inhibition of Fas-mediated $\mathrm{CD8}^{+} \mathrm{T}$ cell cytotoxicity depends on Met, PI3Ka and FLIP}

The receptor of InlB is c-Met ${ }^{24}$, a member of the receptor tyrosine kinases family, which is ubiquitously expressed, including in monocytes ${ }^{25}$. In $L m$-infected monocytes, bacteria are surrounded by LAMP-1, and InlB induces the recruitment of its receptor c-Met, which can be detected around bacteria (Extended Data Fig. 9a). InlB association to bacterial surface mediates the recruitment of c-Met around bacteria, in contrast to InlB released form, that as expected does not recruits c-Met, and does not mediate neuroinvasion (Extended Data Fig. 8d and 9e). Consistent with a critical role of c-Met, its competitive inhibition by capmatinib fully abrogates InlB-mediated neuroinvasion (Fig. 4a and Extended Data Fig. 9f). In mice where c-Met is conditionally deleted in myeloid cells $\left(L y s M-\mathrm{CreER}^{\mathrm{T} 2} \times M e^{f l o x /+}\right.$ or $\left.\times M e t^{f l o x f f l o x}\right)$, InlB-mediated neuroinvasion is also abrogated (Fig. 4b, c). c-Met signals through PI3-kinase (PI3K), leading to the phosphorylation of $\mathrm{Akt}^{26,27}$. Consistent with InlB-mediated c-Met engagement in infected monocytes, Akt is phosphorylated in an InlB-dependent manner in these cells (Extended Data 
Fig. 10a, b). Moreover, inhibition of PI3K activity by the pan-inhibitor wortmannin fully blocks InlB-mediated neuroinvasion (Fig. 4d and Extended Data Fig. 9c). Specifically, inhibition of $\mathrm{PI} 3 \mathrm{~K} \alpha$, but not of the leucocyte-specific PI3K $\delta$, fully abrogates InlB-mediated neuroinvasion (Fig. 4e and Extended Data Fig. 10d, e). FLIP, a PI3K-regulated competitive inhibitor of procaspase- $8^{28,29}$, is upregulated in infected monocytes, in an InlB-dependent manner, resulting in a decreased activity of caspase-8 (Fig. 4f, g and Extended Data Fig. 10f). Inhibition of either c-Met or PI3K $\alpha$ blocks both InlB-mediated FLIP upregulation and the decrease of caspase- 8 activity (Fig. 4f, g). In mice conditionally deleted for FLIP, InlB-mediated monocytes resistance to cell death is lost (Fig. 4h and Extended Data Fig. 10g). Moreover, in mice where FLIP is conditionally deleted in myeloid cells $\left(L y s M-\mathrm{CreER}^{\mathrm{T} 2} \times F L I P^{\text {flox/+ }}\right.$ or $\left.\times F L I P^{\text {floxflox }}\right)$, InlB involvement in neuroinvasion is fully abrogated (Fig. 4i, j). Altogether, these results demonstrate that InlB-mediated blockade of the Fas cell death pathway in infected monocytes results from the PI3K $\alpha$-dependent cell autonomous upregulation of the caspase- 8 inhibitor FLIP (Extended Data Fig. 10h).

\section{InlB inhibition of $\mathrm{CD8}^{+} \mathrm{T}$ cell mediated killing promotes $\mathrm{Lm}$ persistence and fecal carriage} InlB is part of $L m$ core genome and is under purifying selection ${ }^{30,31}$, suggesting that InlB confers a selective advantage to $L m$. As $L m$ is shed back from infected tissues into the intestinal lumen $^{32}, L m$ increased virulence may translate into increased fecal shedding, thereby favoring transmission. We therefore tested whether InlB is also involved in $L m$ intestinal carriage and release in the feces. Indeed, WT- $L m$ levels of infection of intestinal tissues and release in the intestinal lumen and feces are significantly higher than that of $\operatorname{Lm\Delta inlB}$, and these differences, as for neuroinvasion, are fully dependent on $\mathrm{CD} 8^{+} \mathrm{T}$-cells (Fig. 4k, 1). These observations highlight that InlB-mediated neuroinvasion, although resulting from a very specific interference 

within-host persistence and inter-host transmission (Fig 4m). 


\section{Discussion}

Here we uncovered that $\mathrm{Lm}$ is able to render its host cell resistant to $\mathrm{CD} 8^{+}$T-cells-mediated killing. Selective blockade of the Fas-FasL death pathway in infected monocytes allows these cells to survive longer in the blood, and ultimately transfer $L m$ more abundantly to the brain. Importantly, this novel, specific and unanticipated mechanism that creates an intracellular protected niche for $\mathrm{Lm}$ is also involved in its persistence in the intestinal tissue and release in the environment. This mechanism is mediated by the surface protein InlB, which was so far described as involved in $L m$ internalization into non-phagocytic cells, whereas we uncover here its key role as an immunomodulatory protein. InlB promotes monocytes survival and neuroinvasion through a refined and unsuspected mechanism: the upregulation, via a cMet/PI3K $\alpha$-dependent pathway, of the anti-apoptotic factor FLIP, which competitively inhibits caspase- 8 cleavage and blocks Fas-FasL mediated cell death.

This study highlights the critical role played by cellular immunity against intracellular pathogens' neuroinvasion from a microbial perspective. Indeed, whereas extracellular pathogens rely on the binding to specific host cell receptors ${ }^{33}$ or the breaching of host barriers ${ }^{34}$ to invade the CNS, the facultative intracellular pathogen $L m$ takes advantage of its ability to invade and persist within host cells to access to the brain. $L m$ intracellular persistence mediated by InlB promotes $\mathrm{Lm}$ neuroinvasion via ActA-dependent transfer of $\mathrm{Lm}$ from infected monocytes to brain endothelial cells. These results show that the capacity of microbes to survive into cells is a key pathogenic determinant favoring within-host dissemination and ultimately neuroinvasion. Other neuroinvasive intracellular pathogens, such as Mycobacterium tuberculosis and Toxoplasma gondii, also stimulate $\mathrm{PI} 3 \mathrm{~K}^{35,36}$ and survive in myeloid cells. As $L m$, these neuroinvasive microorganisms may also protect infected cells from killing by the immune system, favoring their survival, and increasing their within-host persistence and neuroinvasiveness. 
Intracellular pathogens tend to interfere with innate immune responses to establish a successful infection, and some also interfere with the adaptive immune system in a broad and non-selective manner ${ }^{37,38}$, favoring their persistence, like HIV, EBV and measles virus. $L m$ has been instrumental for the discovery of cellular immunity ${ }^{39}$ and is indeed a prototypic inducer of a protective $\mathrm{CD}^{+} \mathrm{T}$-cell response $\mathrm{e}^{20,40}$. Yet, we have shown that InlB selectively blocks the action of the most efficient and specific anti- $\mathrm{Lm}$ immune effector, T cells-mediated cytotoxicity. This allows the establishment of a protected niche favoring $L m$ dissemination and persistence within the host. This is reminiscent of the mechanism by which tumor cells, in which signaling downstream of growth factor receptors is frequently constitutively activated ${ }^{41,42}$, also evade immune responses by surviving immune killing. A detailed understanding of how microbes have selected mechanisms to interfere with the immune system may help to rationally design novel anti-infective and anti-tumor therapies. Similarly, the immunomodulatory mechanism of InlB, specific of and restricted to infected cells, may also help develop new immunosuppressive therapies aimed at specifically protecting cells of interest from the immune system, as opposed to classic immunosuppressive drugs that inhibit indiscriminately immune functions, and therefore favor infectious and neoplastic complications.

$L m$ is an opportunistic pathogen that only rarely induces clinically apparent infection upon oral ingestion ${ }^{43}$, and there is no inter-human transmission of listeriosis. Yet, the so-called "virulence factors" of $L m$ are under purifying selection ${ }^{30,31,44}$, implying that they contribute to $L m$ fitness. By interfering with the host anti- $L m$ cellular effectors, we have shown that InlB enhances $L m$ intestinal carriage and fecal shedding, thereby increasing the odds of neuroinvasive $L m$ to be transmitted back to the environment and colonize new hosts. This illustrates that the anthropocentric view on microbial pathogenesis which phenotypic output is centered on disease does not necessarily reflect the actual context where microbial evolution and fitness gain take place. 


\section{References and Notes:}

1. Schuchat, A. et al. Bacterial Meningitis in the United States in 1995. New England Journal of Medicine 337, 970-976 (1997).

2. van de Beek, D. et al. Clinical Features and Prognostic Factors in Adults with Bacterial Meningitis. New England Journal of Medicine 351, 1849-1859 (2004).

3. Charlier, C. et al. Clinical features and prognostic factors of listeriosis: the MONALISA national prospective cohort study. The Lancet Infectious Diseases 17, 510-519 (2017).

4. Mailles, A. \& Stahl, J. Infectious Encephalitis in France in 2007: A National Prospective Study. Clinical Infectious Diseases 49, 1838-1847 (2009).

5. Skogberg, K. et al. Clinical Presentation and Outcome of Listeriosis in Patients with and without Immunosuppressive Therapy. Clinical Infectious Diseases 14, 815-821 (1992).

6. Maury, M. M. et al. Uncovering Listeria monocytogenes hypervirulence by harnessing its biodiversity. Nature Genetics 48, 308-313 (2016).

7. Disson, O. et al. Conjugated action of two species-specific invasion proteins for fetoplacental listeriosis. Nature 455, 1114-1118 (2008).

8. Drevets, D. A., Jelinek, T. A. \& Freitag, N. E. Listeria monocytogenes-infected phagocytes can initiate central nervous system infection in mice. Infection and Immunity 69, 1344-1350 (2001).

9. Join-Lambert, O. F. et al. Listeria monocytogenes-infected bone marrow myeloid cells promote bacterial invasion of the central nervous system. Cellular microbiology 7 , 167-180 (2005).

10. Cantinelli, T. et al. "Epidemic clones" of Listeria monocytogenes are widespread and ancient clonal groups. Journal of clinical microbiology 51, 3770-9 (2013).

11. Bécavin, C. et al. Comparison of widely used Listeria monocytogenes strains EGD, 10403S, and EGD-e highlights genomic variations underlying differences in pathogenicity. mBio 5, e00969-14 (2014).

12. Boring, L. et al. Impaired monocyte migration and reduced type 1 (Th1) cytokine responses in C-C chemokine receptor 2 knockout mice. Journal of Clinical Investigation 100, 2552-2561 (1997).

13. Kocks, C. et al. L. monocytogenes-induced actin assembly requires the actA gene product, a surface protein. Cell 68, 521-531 (1992).

14. Tilney, L. G. \& Portnoy, D. A. Actin filaments and the growth, movement, and spread of the intracellular bacterial parasite, Listeria monocytogenes. The Journal of cell biology 109, 1597-1608 (1989).

15. Lecuit, M. et al. A transgenic model for listeriosis: Role of internalin in crossing the intestinal barrier. Science 292, 1722-1725 (2001).

16. Gaillard, J. L., Jaubert, F. \& Berche, P. The inlAB locus mediates the entry of Listeria monocytogenes into hepatocytes in vivo. The Journal of experimental medicine 183, 359-369 (1996).

17. Braun, L. et al. InlB: an invasion protein of Listeria monocytogenes with a novel type of surface association. Molecular microbiology 25, 285-294 (1997).

18. Lane, F. C. \& Unanue, E. R. Requirement of thymus (T) lymphocytes for resistance to listeriosis. The Journal of experimental medicine 135, 1104-1112 (1972).

19. Schaffner, A., Douglas, H. \& Davis, C. E. Models of T cell deficiency in listeriosis: the effects of cortisone and cyclosporin A on normal and nude BALB/c mice. Journal of immunology 131, 450-453 (1983). 
20. Pamer, E. G. Immune responses to Listeria monocytogenes. Nature Reviews Immunology vol. 4 812-823 (2004).

21. Khanna, K. M., McNamara, J. T. \& Lefrançois, L. In situ imaging of the endogenous CD8 T cell response to infection. Science 318, 116-120 (2007).

22. Doherty, P. Cell-mediated cytotoxicity. Cell 75, 607-612 (1993).

23. Muzio, M. et al. FLICE, a novel FADD-homologous ICE/CED-3-like protease, is recruited to the CD95 (Fas/APO-1) death-inducing signaling complex. Cell 85, 817827 (1996).

24. Shen, Y., Naujokas, M., Park, M. \& Ireton, K. InIB-dependent internalization of Listeria is mediated by the Met receptor tyrosine kinase. Cell 103, 501-510 (2000).

25. Beilmann, M. et al. Neoexpression of the c-met/hepatocyte growth factor-scatter factor receptor gene in activated monocytes. Blood 90, 4450-4458 (1997).

26. Bowers, D. C. et al. Scatter factor/hepatocyte growth factor protects against cytotoxic death in human glioblastoma via phosphatidylinositol 3-kinase- and AKT-dependent pathways. Cancer Research 60, 4277-4283 (2000).

27. Xiao, G.-H. et al. Anti-apoptotic signaling by hepatocyte growth factor/Met via the phosphatidylinositol 3-kinase/Akt and mitogen-activated protein kinase pathways.

Proceedings of the National Academy of Sciences 98, 247-252 (2001).

28. Osaki, M. et al. Inhibition of the PI3K-Akt signaling pathway enhances the sensitivity of Fas-mediated apoptosis in human gastric carcinoma cell line, MKN-45. Journal of Cancer Research and Clinical Oncology 130, 8-14 (2004).

29. Moumen, A. et al. Met signals hepatocyte survival by preventing Fas-triggered FLIP degradation in a PI3K-Akt-dependent manner. Hepatology 45, 1210-1217 (2007).

30. Tsai, Y. H. L., Orsi, R. H., Nightingale, K. K. \& Wiedmann, M. Listeria monocytogenes internalins are highly diverse and evolved by recombination and positive selection. Infection, Genetics and Evolution 6, 378-389 (2006).

31. Moura, A. et al. Whole genome-based population biology and epidemiological surveillance of Listeria monocytogenes. Nature Microbiology 2, 16185 (2016).

32. Louie, A., Zhang, T., Becattini, S., Waldor, M. K. \& Portnoy, D. A. A multiorgan trafficking circuit provides purifying selection of listeria monocytogenes virulence genes. mBio 10, (2019).

33. Coureuil, M., Lécuyer, H., Bourdoulous, S. \& Nassif, X. A journey into the brain: insight into how bacterial pathogens cross blood-brain barriers. Nature Reviews Microbiology 15, 149-159 (2017).

34. Devraj, G. et al. HIF-1 $\alpha$ is involved in blood-brain barrier dysfunction and paracellular migration of bacteria in pneumococcal meningitis. Acta Neuropathologica 140, 183-208 (2020).

35. Liu, Y., Li, J. Y., Chen, S. T., Huang, H. R. \& Cai, H. The rLrp of mycobacterium tuberculosis inhibits proinflammatory cytokine production and downregulates APC function in mouse macrophages via a TLR2-mediated PI3K/Akt pathway activationdependent mechanism. Cellular and Molecular Immunology 13, 729-746 (2016).

36. Quan, J. H. et al. Intracellular networks of the PI3K/AKT and MAPK pathways for regulating Toxoplasma gondii-induced IL-23 and IL-12 production in human THP-1 cells. PLOS ONE 10, e0141550 (2015).

37. Klenerman, P. \& Hill, A. T cells and viral persistence: Lessons from diverse infections. Nature Immunology vol. 6 873-879 (2005).

38. Protzer, U., Maini, M. K. \& Knolle, P. A. Living in the liver: Hepatic infections. Nature Reviews Immunology vol. 12 201-213 (2012).

39. MACKANESS, G. B. Cellular resistance to infection. The Journal of experimental medicine 116, 381-406 (1962). 
40. Shen, H. et al. Recombinant Listeria monocytogenes as a live vaccine vehicle for the induction of protective anti-viral cell-mediated immunity. Proceedings of the National Academy of Sciences 92, 3987-3991 (2006).

41. Spranger, S., Bao, R. \& Gajewski, T. F. Melanoma-intrinsic $\beta$-catenin signalling prevents anti-tumour immunity. Nature 523, 231-235 (2015).

42. Tauriello, D. V. F. et al. TGF $\beta$ drives immune evasion in genetically reconstituted colon cancer metastasis. Nature 554, 538-543 (2018).

43. Ricci, A. et al. Listeria monocytogenes contamination of ready-to-eat foods and the risk for human health in the EU. EFSA Journal 16, (2018).

44. Maury, M. M. et al. Spontaneous Loss of Virulence in Natural Populations of Listeria monocytogenes. Infection and immunity 85, (2017). 


\section{Acknowledgments}

We thank Philippe Bousso and Alain Fischer for helpful discussions, and the members of the Biology of Infection Unit for their support, in particular Laetitia Travier for technical help on brain microscopy and Lukas Hafner for contributing to data analysis. We thank the Cytometry and Biomarkers Unit of Technology and Service (CB UTechS), Dmity Ershov from the Image Analysis Hub and the Center for Animal Resources and Research (C2RA) at Institut Pasteur. We are grateful to Geneviève de Saint Basile and Fernando Sepuvelda (Institut Imagine, Paris) for the Prf1 KO mice, Frédéric Rieux-Laucat (Institut Imagine, Paris) for the Fas ${ }^{\text {lpr-cg }}$ mice, Richard Pope for the FLIP flox/flox mice, Florian Greten for the LysMCreER ${ }^{\mathrm{T} 2}$ mice, Alain Eychene for the Met ${ }^{\text {flox/flox }}$ mice, and Javier Pizarro-Cerda and Pascale Cossart (Institut Pasteur) for the pAD $\beta$-lactamase plasmid. Funding: Work in ML laboratory is funded by Institut Pasteur, Inserm, ERC, ANR and Labex IBEID (ANR-10-LABX-62-IBEID). CM was a recipient of the Roux-Cantarini fellowship of Institut Pasteur. LH, CG and JG were supported by Université Paris Descartes, YHT by the Pasteur - Paris University (PPU) International PhD Program, under the European Union's Horizon 2020 research and innovation program, Marie Sklodowska-Curie grant agreement No 665807, and SL by FRM (ECO201906009119) and "Ecole Doctorale FIRE-Programme Bettencourt”. Author contributions: CM, MK, and ML designed the experimental strategy. CM, MK, SL, JG, YHT and OD designed and performed mouse experiments. CM, LH and CG designed and performed in vitro experiments. CM, MK, SL, JG, OD, YHT and ML analyzed the data. CM, SL and ML wrote the manuscript, MK and OD edited it, and all authors agreed on its final version. Competing interests: The authors declare no competing interests. Data and material availability: The datasets generated during and/or analyzed during the current study are available from the corresponding author on reasonable request. 
427 Extended Data:

$428 \quad$ Materials and Methods

429 Extended Data Tables 1-5

430 Extended Data Figures 1-10

431 Extended Data Movie 1

432 Extended References (45-60) 
Figure 1

a

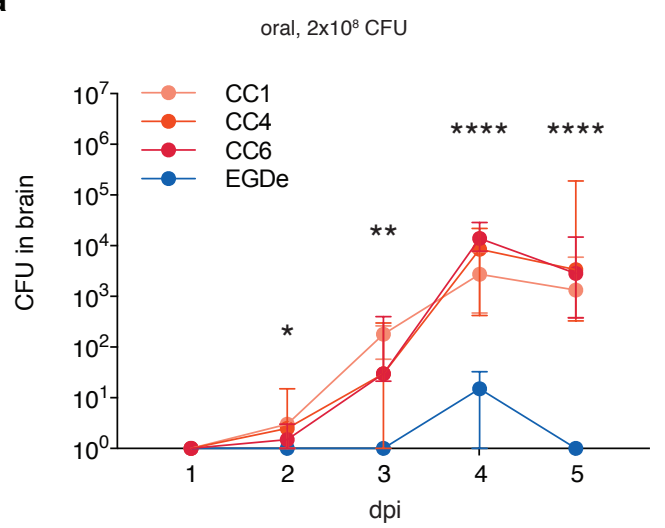

b

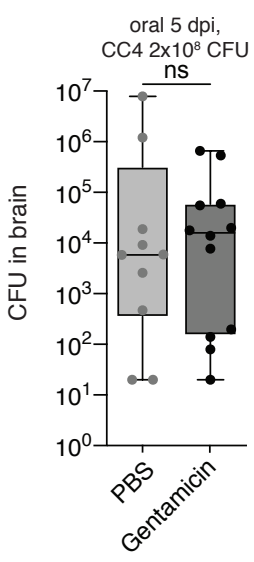

c

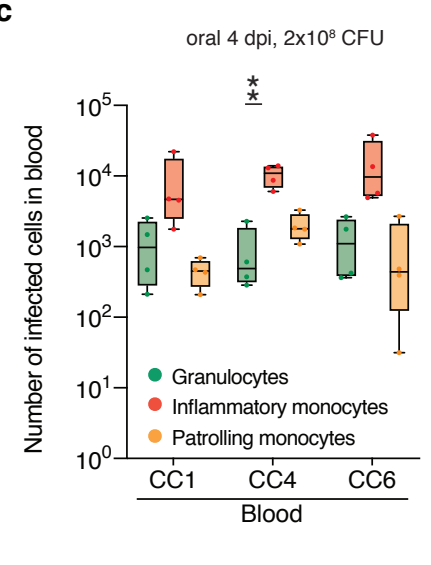

f

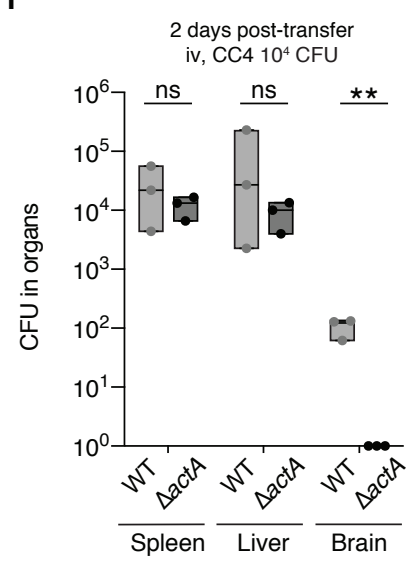

d

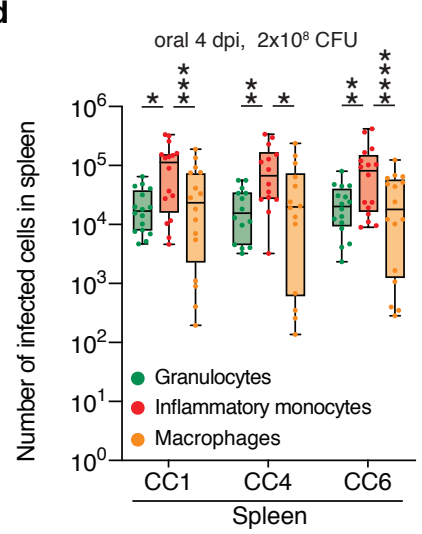

g

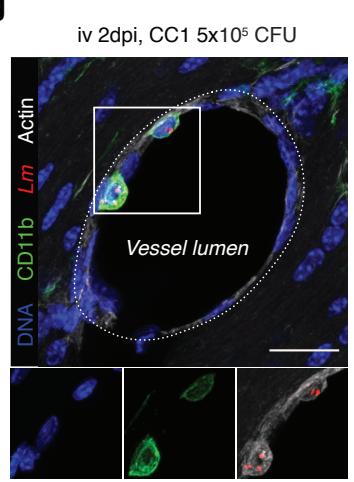

h

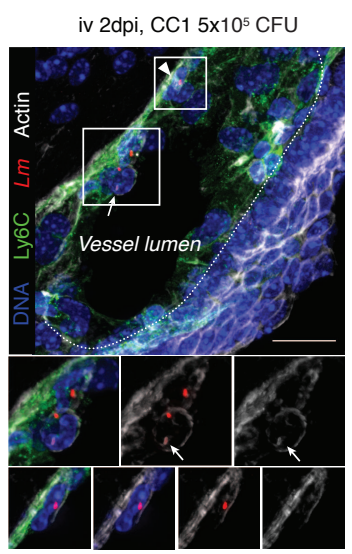

iv, $\mathrm{CC} 410^{4} \mathrm{CFU}$

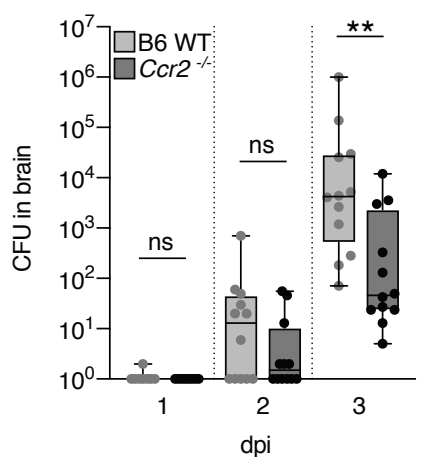

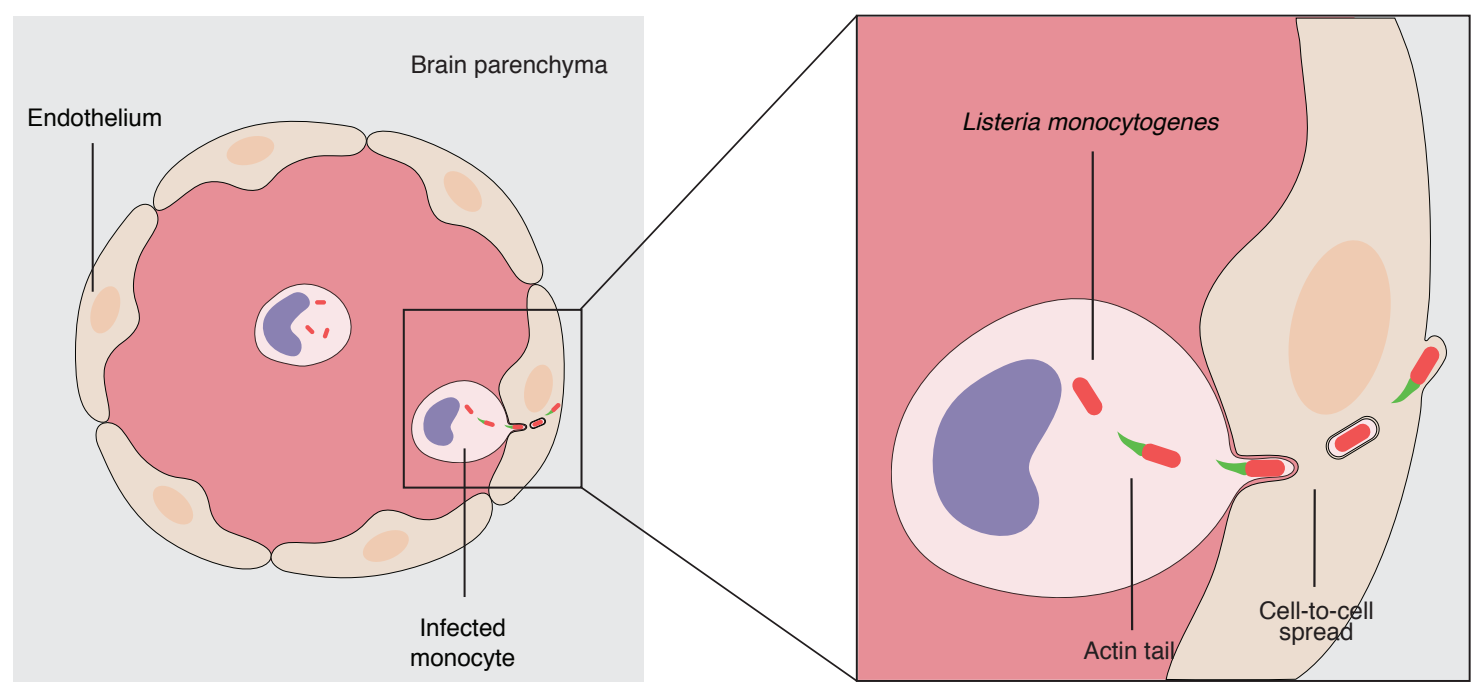


Fig. 1. Infected inflammatory monocytes transfer $L m$ to the CNS by cell-to-cell spread. (a) Bacterial load in the brain of mice after oral inoculation with CC1/CC4/CC6-Lm and EGDe. (b) Bacterial load in the brain of gentamicin-treated mice after oral inoculation with CC4-Lm. (c, d) Three main infected cell subsets in the blood (c) and spleen (d) after oral inoculation with $\mathrm{CC} 1 / \mathrm{CC} 4 / \mathrm{CC} 6-\mathrm{Lm}$ in mice at $4 \mathrm{dpi}$, the peak of bacteremia (Extended Data Fig. 1c). For the blood panel, each dot corresponds to the blood of three mice pooled together. (e) Bacterial load in the brain of B6-WT and $C c r 2^{-/}$mice after intravenous (iv) inoculation with CC4-Lm. (f) Bacterial load of gentamicin-treated recipient mice, 2 days after injection of infected monocytes harvested from 6 donors mice 3 days after iv inoculation with either CC4-WT or CC4AactA. $(\mathbf{g}, \mathbf{h})$ Representative fluorescent microscopy images of infected monocytes adhering to the endothelial cells (g) and of monocytes infected by an actin-polymerizing- $\mathrm{Lm}$ (arrow) adjacent to an infected endothelial cell (arrowhead, h), 2 days after iv inoculation with CC1-Lm. (h) Maximum intensity projection over a $20 \mu \mathrm{m}$ stack and insets are single $z$-planes. Scale bars, $20 \mu \mathrm{m}$. (i) Schematic representation of $L m$ neuroinvasion process. Data were obtained from three (a, b, e and f) and four ( $\mathrm{c}$ and $\mathrm{d}$ ) independent experiments and are presented as median \pm interquartile (a) and as median \pm interquartile (box) and extreme values (lines) (b-f). CFU are compared with the unpaired MannWhitney test ( $a, b$, e and f) and number of infected cells with the Friedman test (c and d). ns: $p>0.05, *: p<0.05,{ }^{* *}: p<0.01,{ }^{* * *}: p<0.001, * * * *: p<0.0001$ 
Figure 2

a

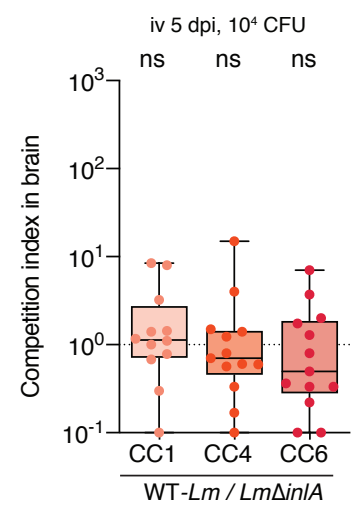

b

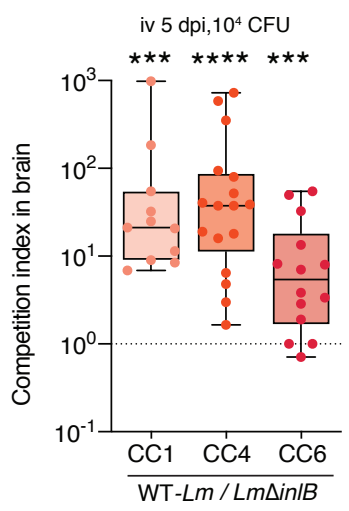

C

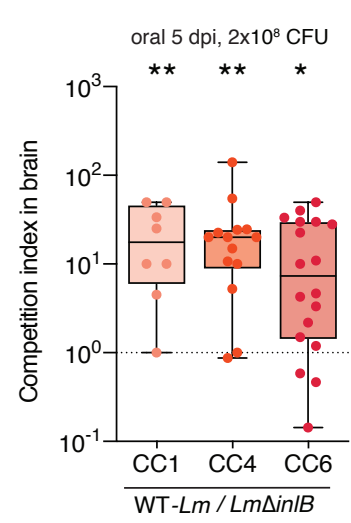

d

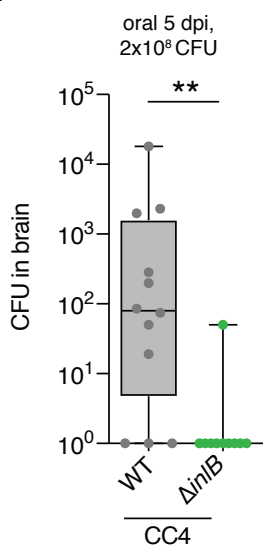

e

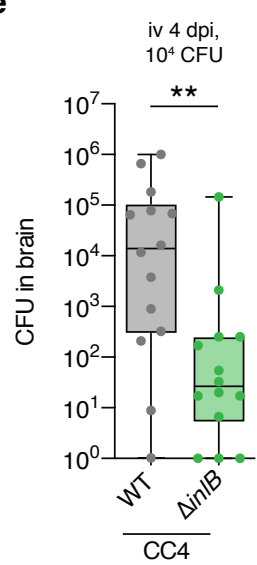

g

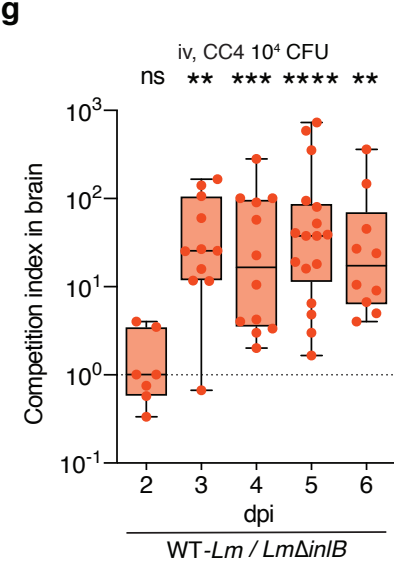

j

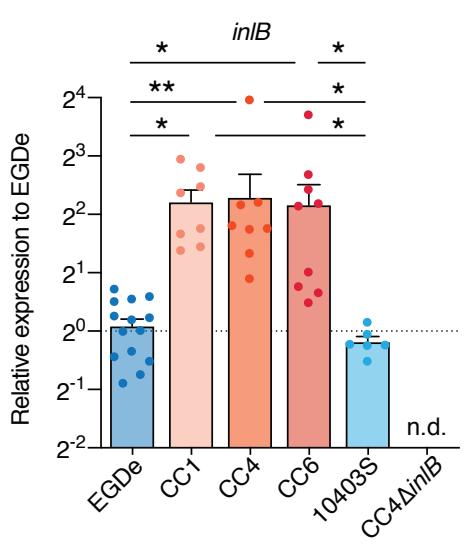

in vivo
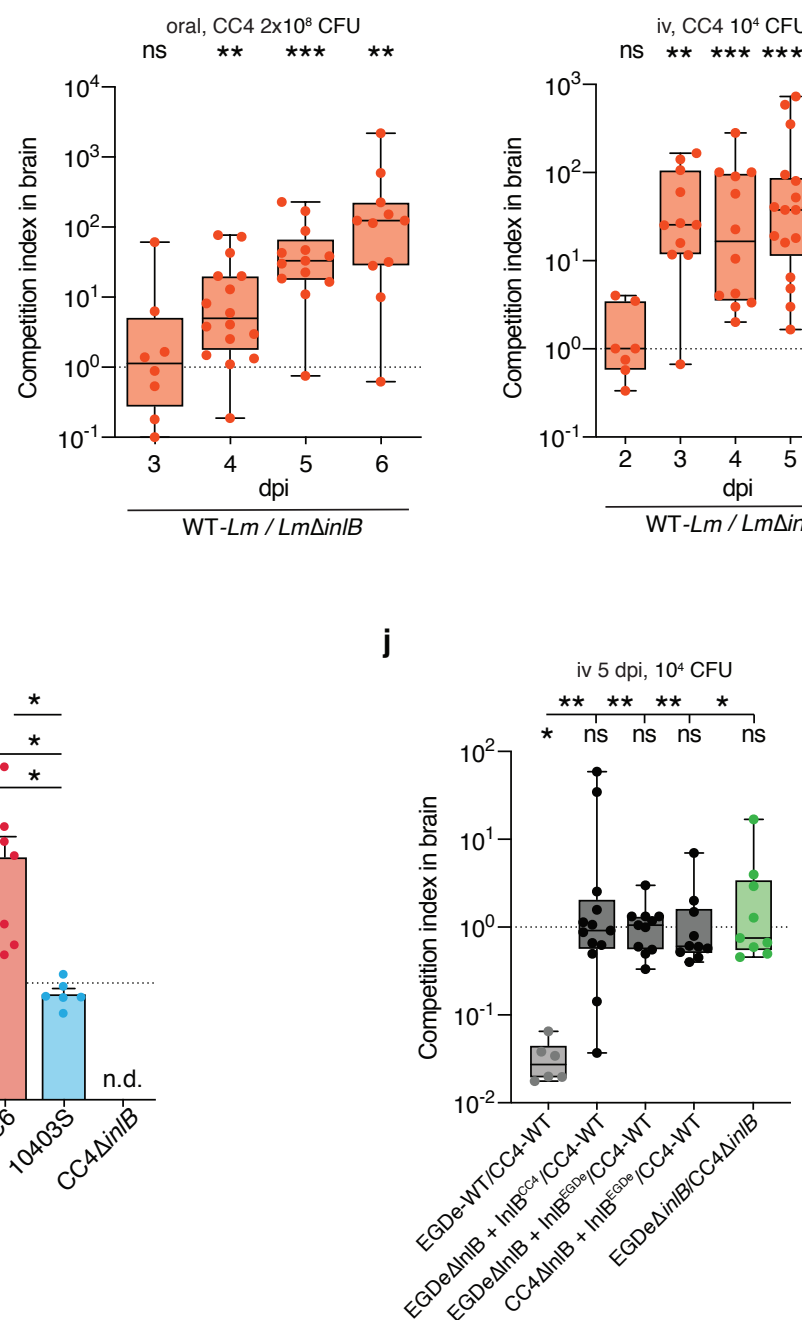

m

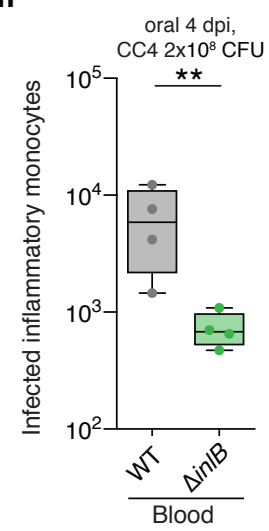

h

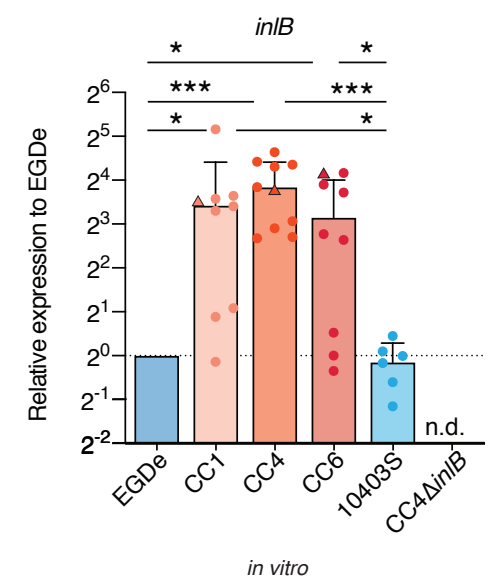

k

iv, CC4 $10^{4} \mathrm{CFU}$

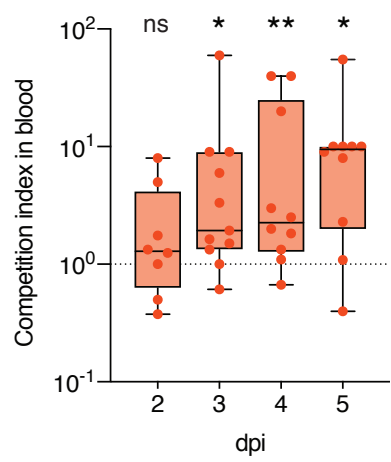

I

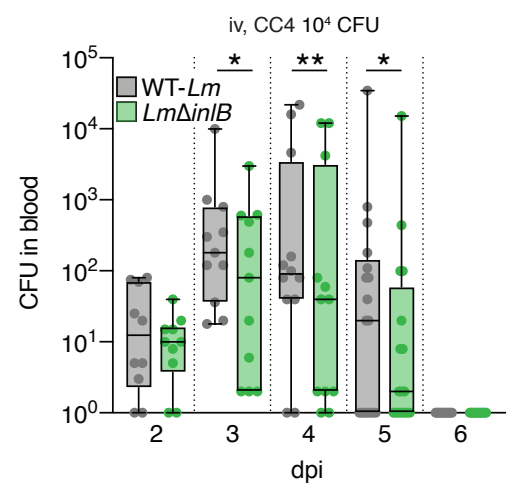

n

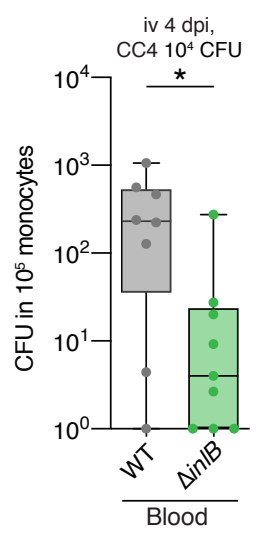

o

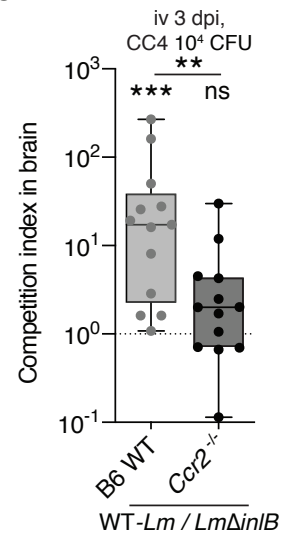


Fig. 2. InlB is involved in $\mathrm{Lm}$ neuroinvasion and inflammatory monocytes infection. (a-c) Competition indexes in brain after $(\mathrm{a}, \mathrm{b})$ iv and after (c) oral inoculation of WT and isogenic mutant strains. (d, e) Bacterial load in brain after (d) oral and (e) iv inoculation with either CC4-WT or CC4AinlB. (f, g) Competition indexes in brain after (f) oral and (g) iv inoculation with 1:1 CC4WT and CC4 $\Delta$ inlB. (h, i) Transcription levels of inlB relative to EGDe in (h) mid-log phase in BHI and (i) in infected splenocytes 2 days after iv inoculation. In (h), each dot for CC1/4/6 corresponds to a different clinical isolate and triangles point out the strains used throughout the rest of the study and referred to as $\mathrm{CC} 1, \mathrm{CC} 4$ and CC6. (j) Competition index in brain after iv inoculation with a 1:1 mix of the indicated bacterial strains $\left(2 \times 10^{4}\right.$ for EGDe $\Delta i n l B$ and CC4 $\left.\operatorname{inl} B\right)$. $(\mathbf{k}, \mathbf{l})$ Competition index (k) and bacterial load (1) in blood after iv inoculation with 1:1 CC4-WT and CC4AinlB. $(\mathbf{m}, \mathbf{n})$ Number of infected monocytes $(\mathrm{m})$ and bacterial enumeration from sorted monocytes (n) in the blood after (m) oral and (n) iv inoculation with CC4-WT or CC4AinlB. (o) Competition index in brain of control or $\mathrm{Ccr}^{-/-}$mice after iv inoculation with 1:1 CC4-WT and CC4 4 inlB. Data were obtained from three (a-h, $\mathrm{j}-1$ and $\mathrm{n}$ ) and four (i, $\mathrm{m}$ and $\mathrm{o}$ ) independent experiments and are presented as median \pm interquartile (box) and extreme values (lines) (a-g and $\mathrm{j}-\mathrm{o}$ ) or as mean $\pm \mathrm{SD}$ (h and $\mathrm{i}$ ). CFU in competition assays are compared with the Wilcoxon matched-pairs signed rank test (a-c, f-g, j-l and o) and samples compared with the unpaired MannWhitney test (d-e, m-o) or the Kruskal-Wallis test (h-j). ns: $p>0.05, *: p<0.05, * *: p<0.01, * * *$ : $p<0.001, * * * *: p<0.0001$. 
Figure 3

a

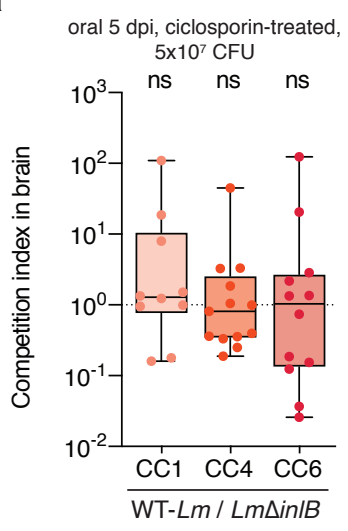

b

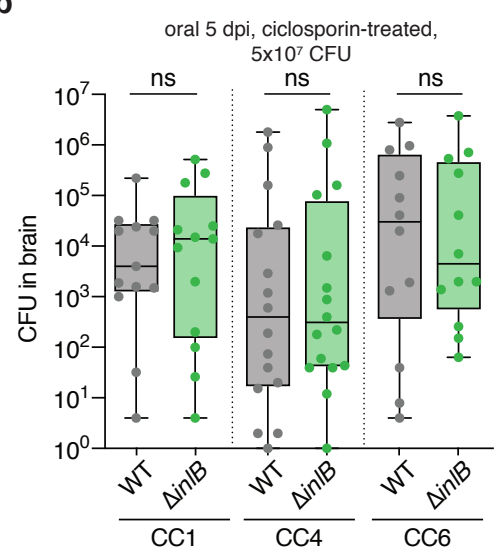

f

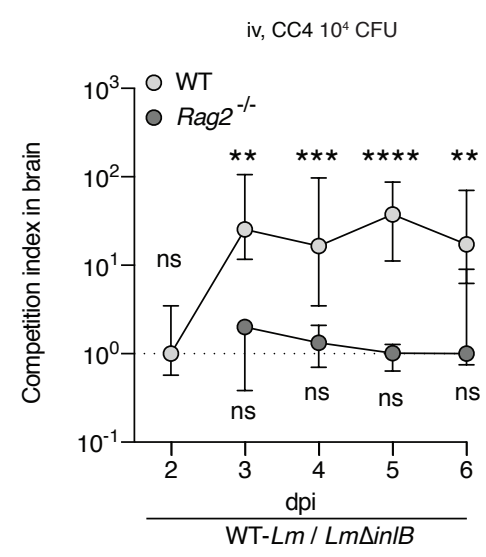

c

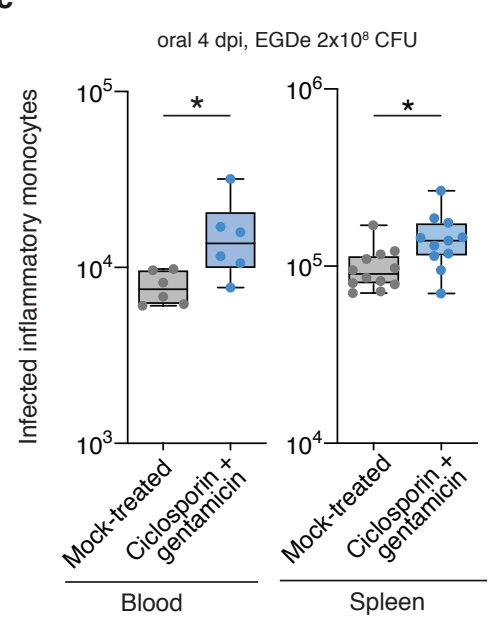

g

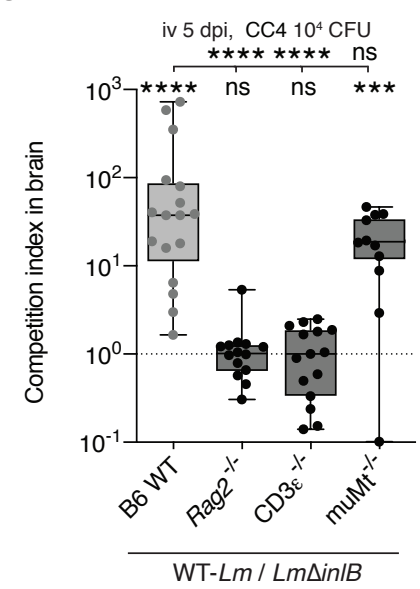

d

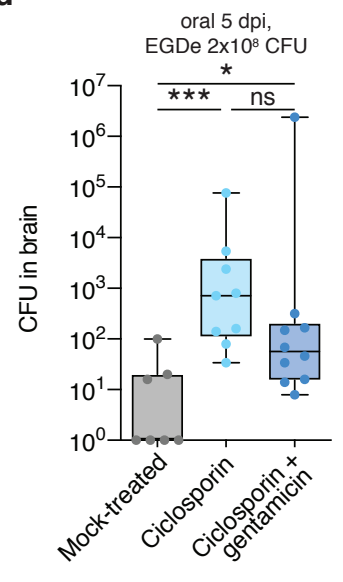

h

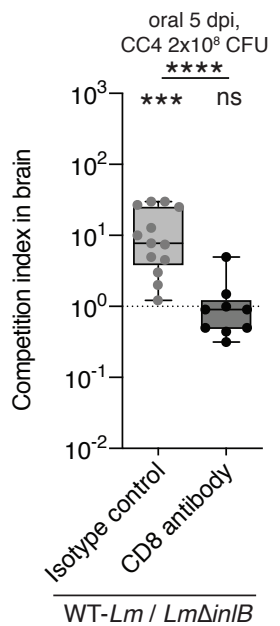

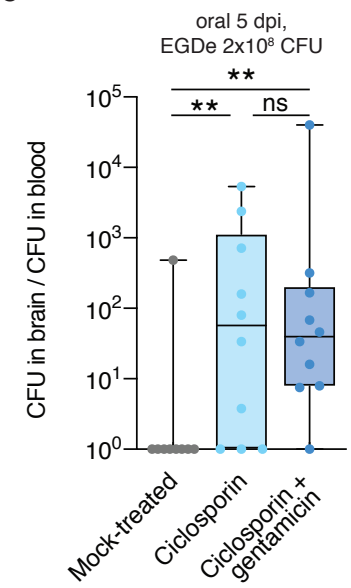

Monocyte

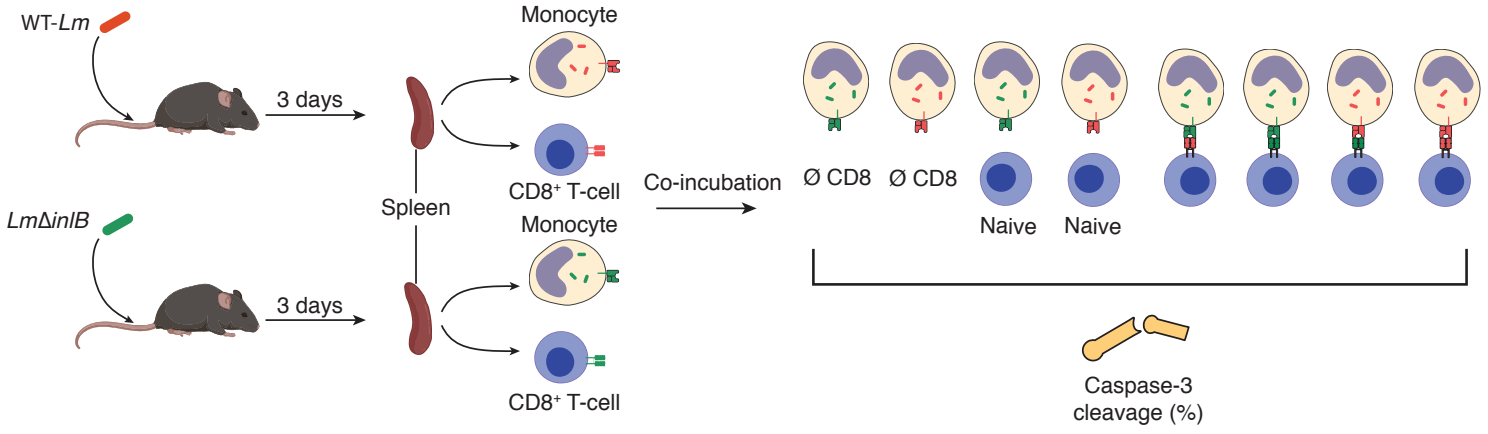

j

iv $3 \mathrm{dpi}, \mathrm{CC} 410^{4} \mathrm{CFU}$

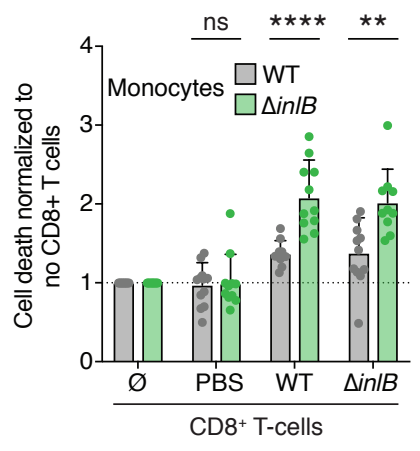

k

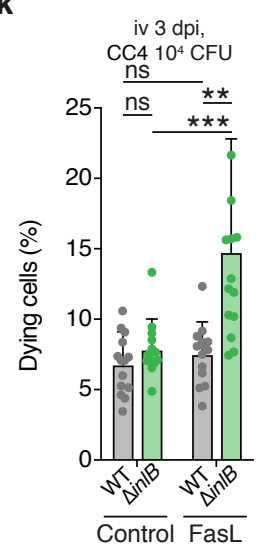

I

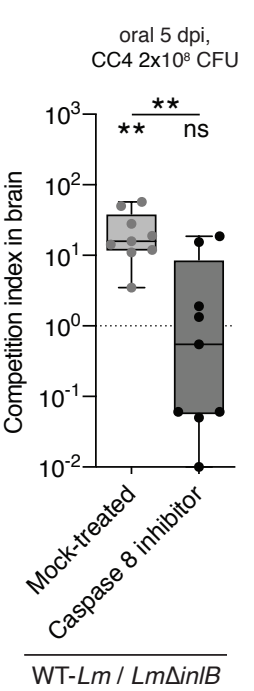

m

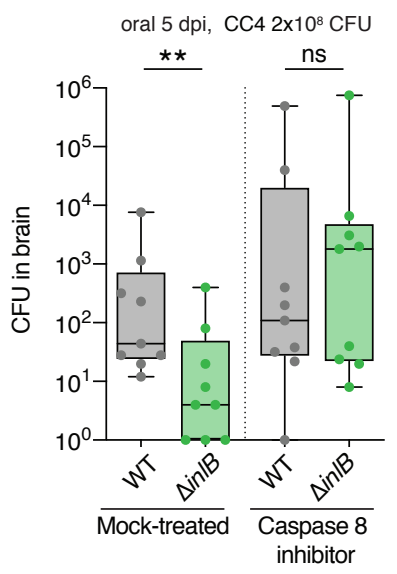


Fig. 3. InIB blocks $\mathrm{CD8}^{+} \mathrm{T}$-cells-mediated monocyte cell death. (a, b) Competition index (a) and bacterial load (b) in brain after inoculation with 1:1 WT- $L m$ strain and $\triangle$ inlB isogenic mutant in ciclosporin-treated mice, related to Fig. 2c. (c) Number of infected monocytes of ciclosporin and gentamicin-treated mice after inoculation with EGDe. (d, e) Bacterial load in brain (d) and ratio of brain/blood bacterial load (e) in ciclosporin \pm gentamicin-treated mice after inoculation with EGDe. (f) Competition index in brain after inoculation with 1:1 CC4-WT strain and CC4 4 inlB in control and $R a g 2^{--}$mice. (g) Competition index in brain after inoculation with 1:1 CC4-WT strain and CC4 4 inlB isogenic mutant in control and in mice lacking functional T (CD3 $\varepsilon^{-}$ $\left.{ }^{\prime}\right)$, B lymphocytes $\left(\mathrm{muMt}^{-/-}\right)$or both $\left(\operatorname{Rag}^{-/}\right)$. (h) Competition index in brain after inoculation with 1:1 CC4-WT and CC4 $\Delta$ inlB after T-CD8 ${ }^{+}$depletion. (i) Schematic pipeline of the cytotoxic lymphocyte (CTL) assay. (j, k) Level of caspase-3 cleavage of infected spleen monocytes, harvested after inoculation with CC4-WT or CC4 $\operatorname{CinlB}$, and incubated with $\mathrm{CD} 8^{+} \mathrm{T}$-cells from similarly infected (WT and $\Delta$ inlB) or control (PBS) mice at an effector to target ratio of $5(\mathrm{j})$ or treated ex vivo with FasL (k). (l, m) Competition index (l) and bacterial load (m) in the brain after inoculation with 1:1 CC4-WT and CC4 $\triangle$ inlB and after treatment with caspase-8 inhibitor. Data were obtained from three (a-h, 1-m) and four (j-k) independent experiments and are presented as median \pm interquartile (box) and extreme values (lines) (a-h, l-m) and as mean $\pm \mathrm{SD}(\mathrm{j}-\mathrm{k}) . \mathrm{CFU}$ in competition assays are compared with the Wilcoxon matched-pairs signed rank test (a-b, f-h, l-m), samples are compared with the Mann-Whitney test (c-e, h, 1-m), an unpaired student $t$-test (j-k) and the Kruskal-Wallis test (g). ns: $p>0.05, *: p<0.05,{ }^{* *}: p<0.01, * * *: p<0.001,{ }^{* * * *}: p<0.0001$. 
Figure 4

a

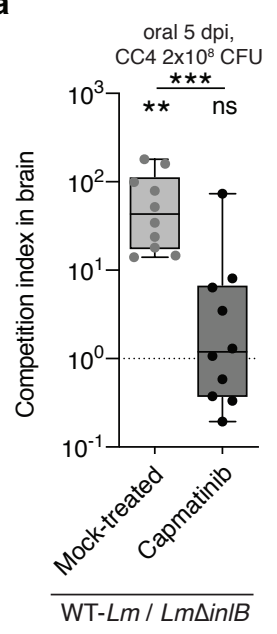

b

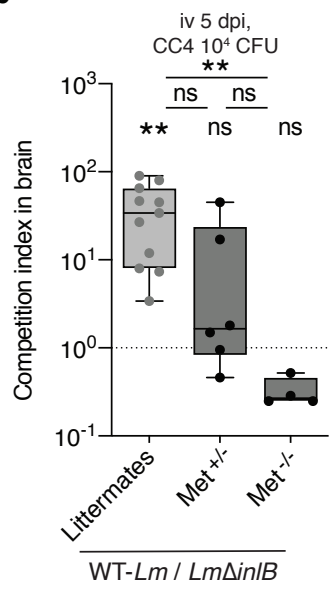

c

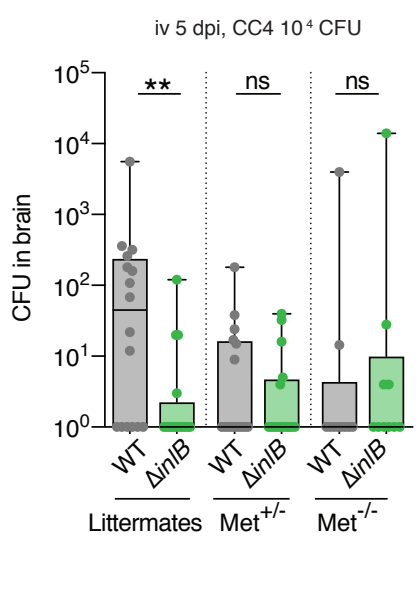

d

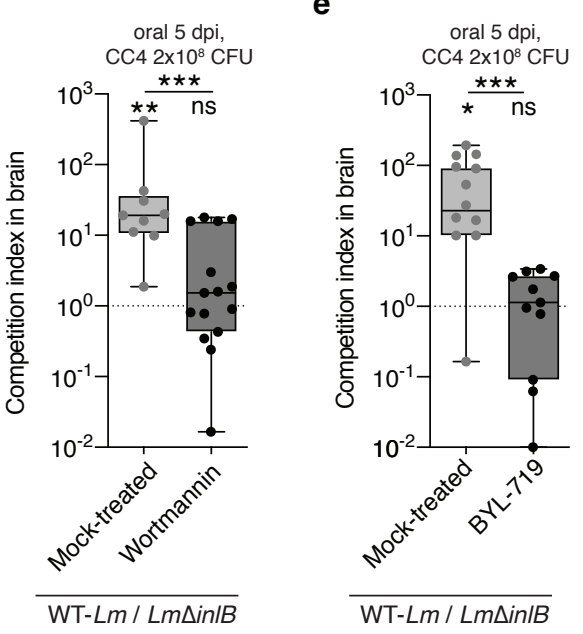

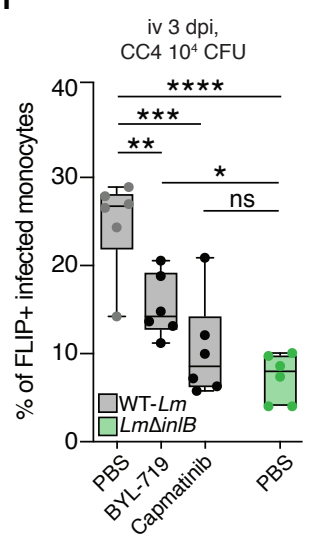

j

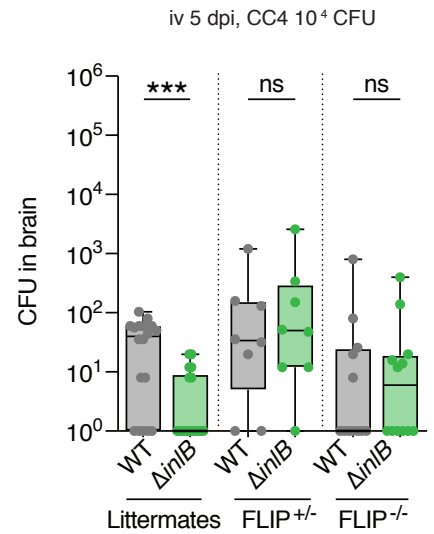

g

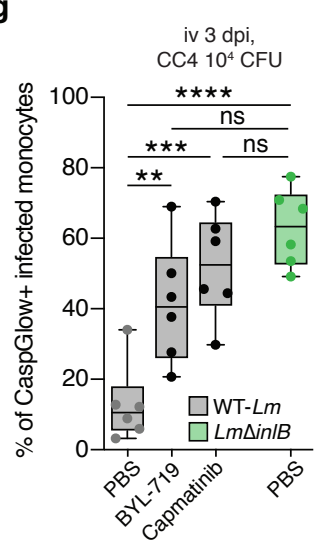

h

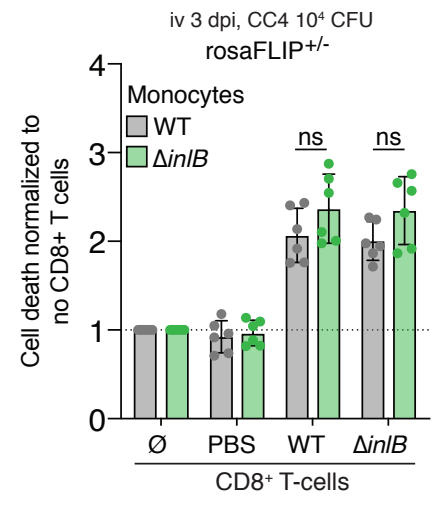

i

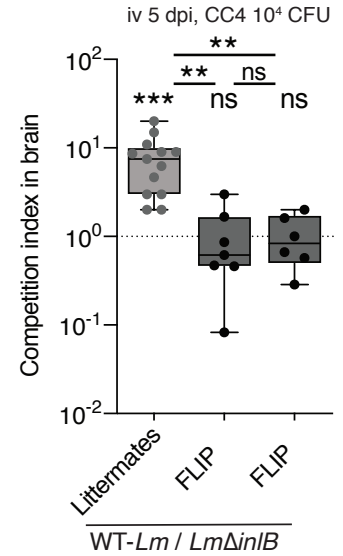

k

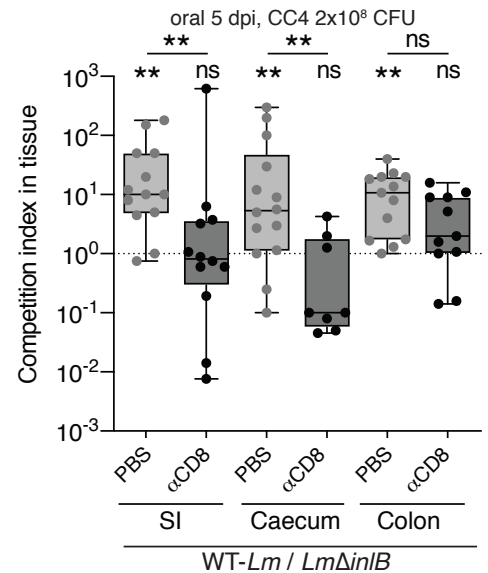

I

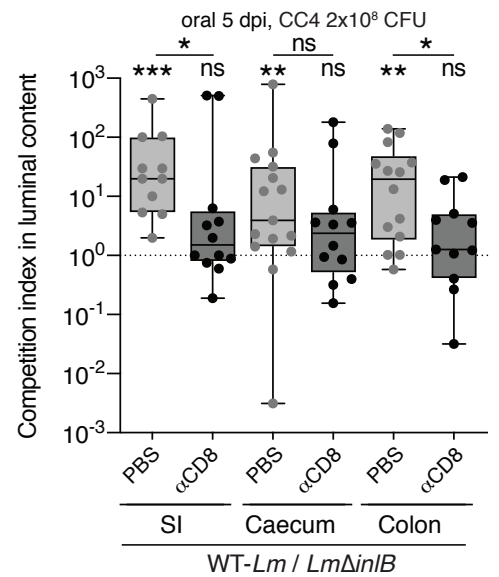

m

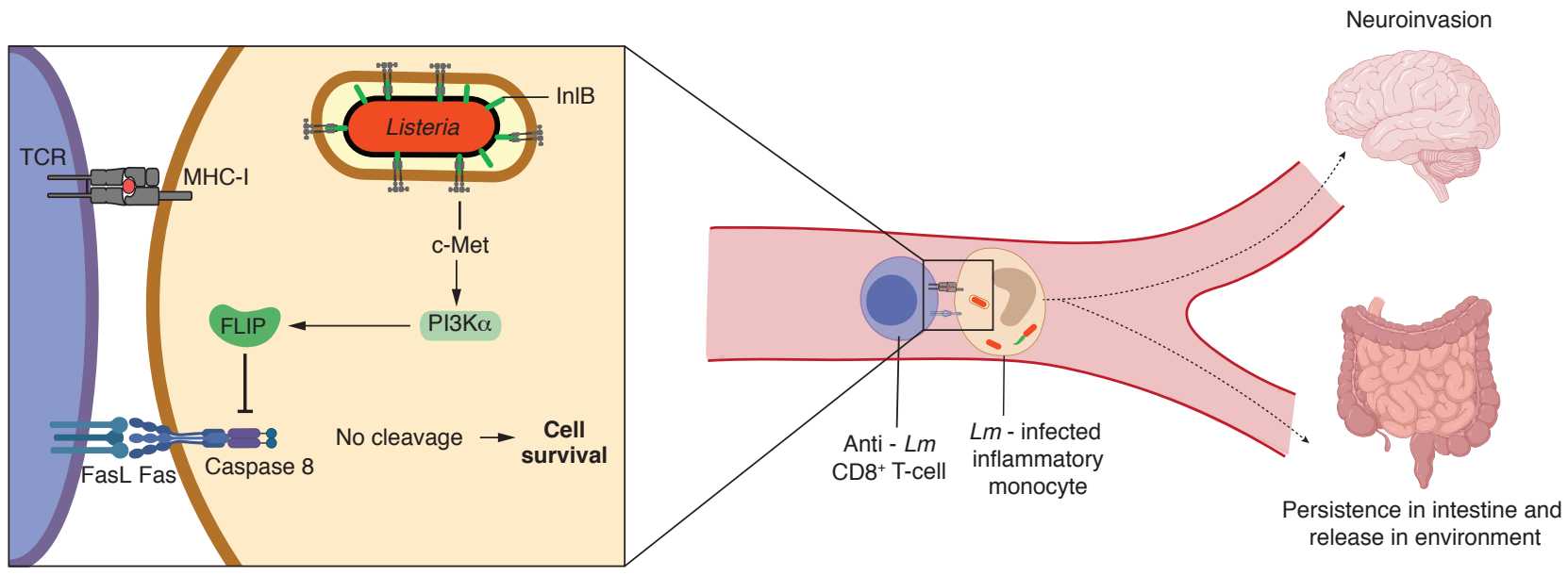


Fig. 4. Inhibition of $\mathrm{CD8}^{+} \mathrm{T}$ cells-mediated cell death by InIB is dependent on the c-MetPI3K $\alpha$-FLIP pathway and is involved in $\mathbf{L m}$ intestinal colonization and fecal carriage. (a) Competition index in the brain after inoculation with 1:1 of CC4-WT and CC4 $\triangle i n l B$, in mice treated with a c-Met inhibitor (capmatinib). (b, c) Competition index (b) and bacterial load (c) in the brain of $L y s M-\mathrm{CreER}^{\mathrm{T} 2} \times \mathrm{Met}^{\text {flox/flox }}$ (or $\mathrm{Met}^{+/ f l o x}$ ) mice (referred to as $\mathrm{Met}^{-/ /}$and $\mathrm{Met}^{+/-}$mice), and their littermates, after inoculation with 1:1 of CC4-WT and CC4AinlB and tamoxifen treatment. (d, e) Competition indexes in the brain after inoculation with 1:1 of CC4-WT and $\mathrm{CC} 4 \Delta i n l B$, in mice treated with a pan-PI3K inhibitor (wortmannin, d) or a specific PI3K $\alpha$ inhibitor (BYL-719, e). (f, g) Proportion of infected monocytes expressing FLIP (f) or active caspase-8 (g) after inoculation with CC4-WT and CC4 $\triangle$ inlB in mice treated with either BYL-719 or capmatinib. (h) Level of caspase-3 cleavage of infected spleen monocytes, harvested after inoculation with CC4-WT or CC4 4 inlB of tamoxifen-treated Rosa26-CreER ${ }^{\mathrm{T2}} \times \mathrm{Cflar}^{+/ \text {flox }}\left(\right.$ rosaFLIP $\left.^{+/}\right)$mice and incubated with $\mathrm{CD} 8^{+} \mathrm{T}$ cells from similarly infected mice at an effector to target ratio of 5. (i, $\left.\mathbf{j}\right)$ Competition index (i) and bacterial load (j) in $L y s M-\mathrm{CreER}^{\mathrm{T} 2} \times \mathrm{FLIP}^{\text {flox/flox }}\left(\right.$ or $\mathrm{FLIP}^{+/ \text {flox }}$ ) mice (referred to as $\mathrm{FLIP}^{-/-}$and $\mathrm{FLIP}^{+/-}$mice), and their littermates, after inoculation with 1:1 of CC4WT and CC4 $\triangle$ inlB and tamoxifen treatment. $(\mathbf{k}, \mathbf{l})$ Competition index in the intestinal tissues $(\mathrm{k})$ and content (1) after inoculation with 1:1 CC4-WT and CC4 4 inlB in mice treated with an anti$\mathrm{CD}^{+}$T-cells antibody, $\mathrm{SI}=$ small intestine. $(\mathbf{m})$ Schematic representation of InlB-mediated blockade of $\mathrm{CD} 8+\mathrm{T}$ cell-mediated cell death resulting in neuroinvasion, persistence in the intestine and transmission. Data were obtained from three (f-h, k-1) or four (a-e, i-j) independent experiments and are presented as median \pm interquartile (box) and extreme values (lines) (a-g, i-l) or mean $\pm \mathrm{SD}(\mathrm{h})$. CFU in competition assays are compared with the Wilcoxon matched-pairs signed rank test (a-e, i-l) and samples are compared with the Mann-Whitney test (a, d-e, k-1), an 
unpaired student $t$-test (h), a Kruskal-Wallis test (b and i) and a one-way ANOVA (f-g). ns: $p>0.05$, $*: p<0.05, * *: p<0.01, * * *: p<0.001, * * * *: p<0.0001$ 
Figures
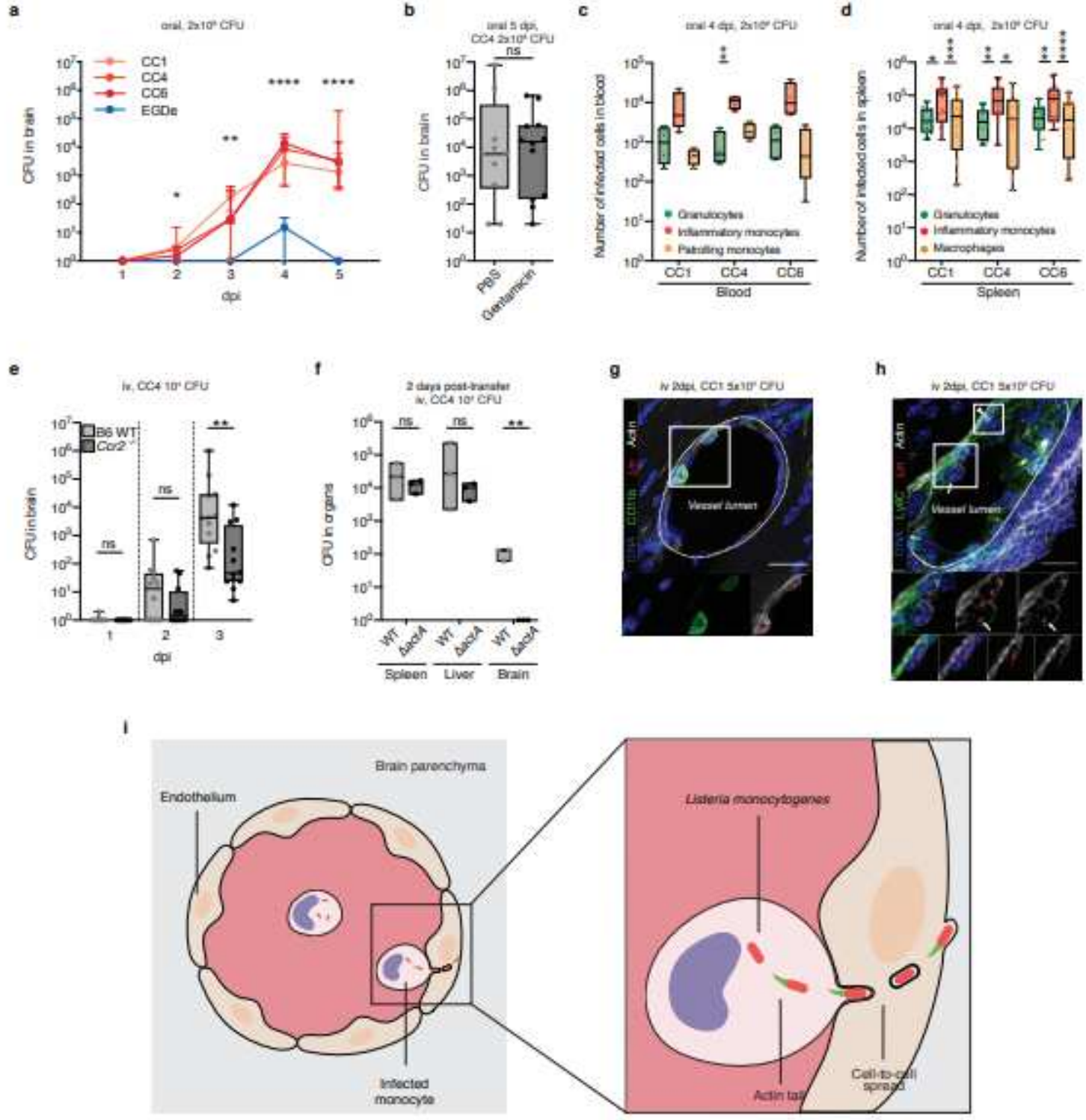

Figure 1

Infected inflammatory monocytes transfer Lm to the CNS by cell-to-cell spread. 
a

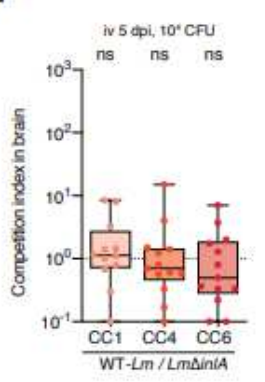

b

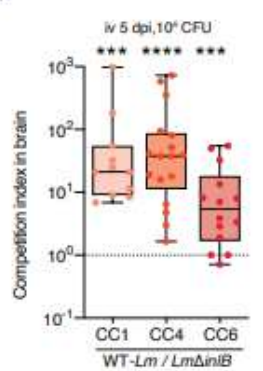

c

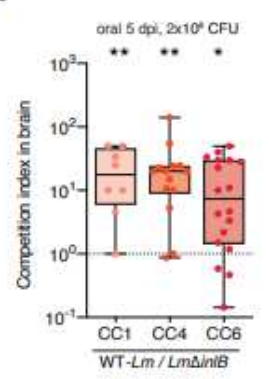

d

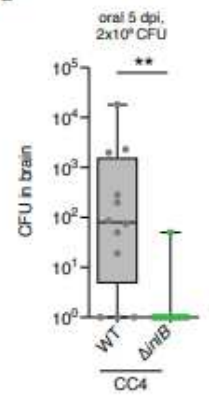

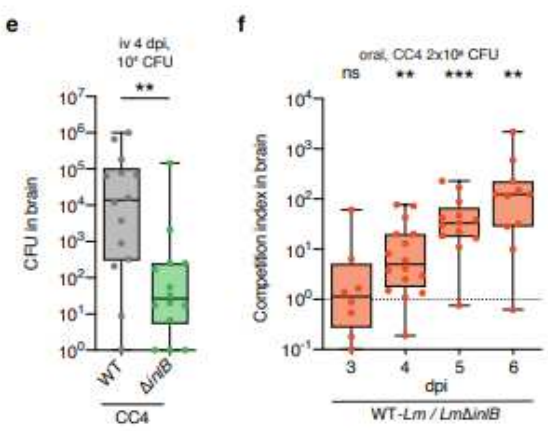

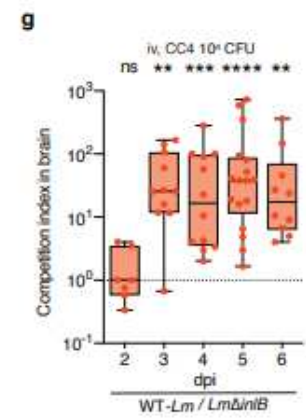

h

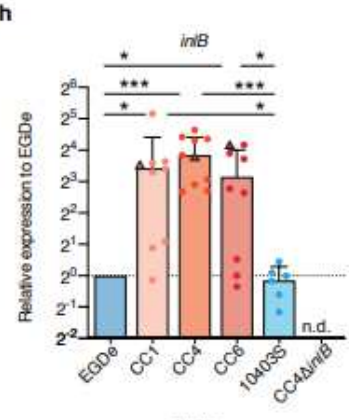

i

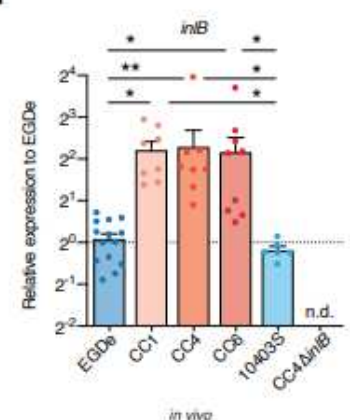

1

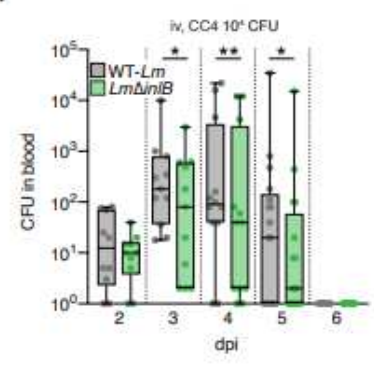

i
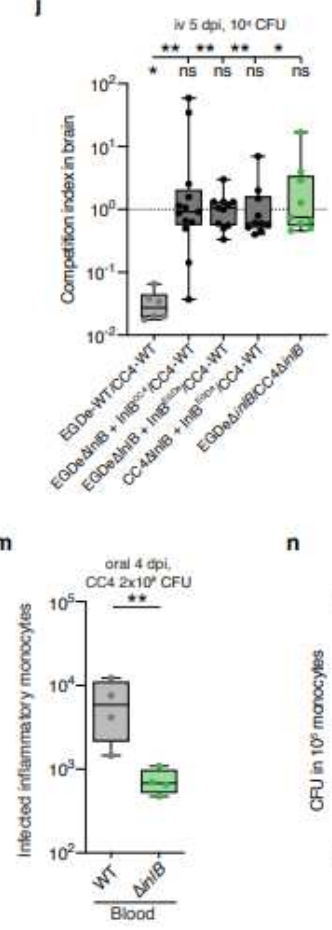

n

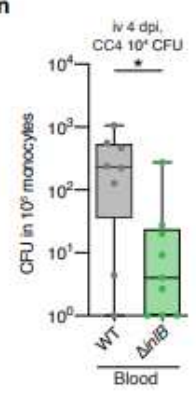

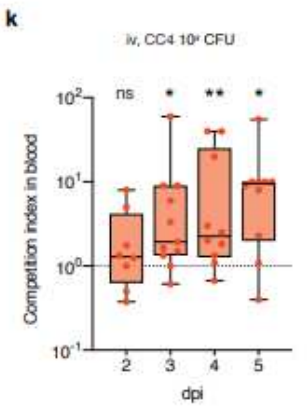

$\circ$

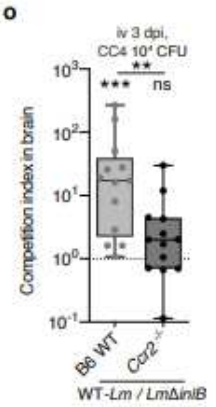

Figure 2

InIB is involved in Lm neuroinvasion and inflammatory monocytes infection. 

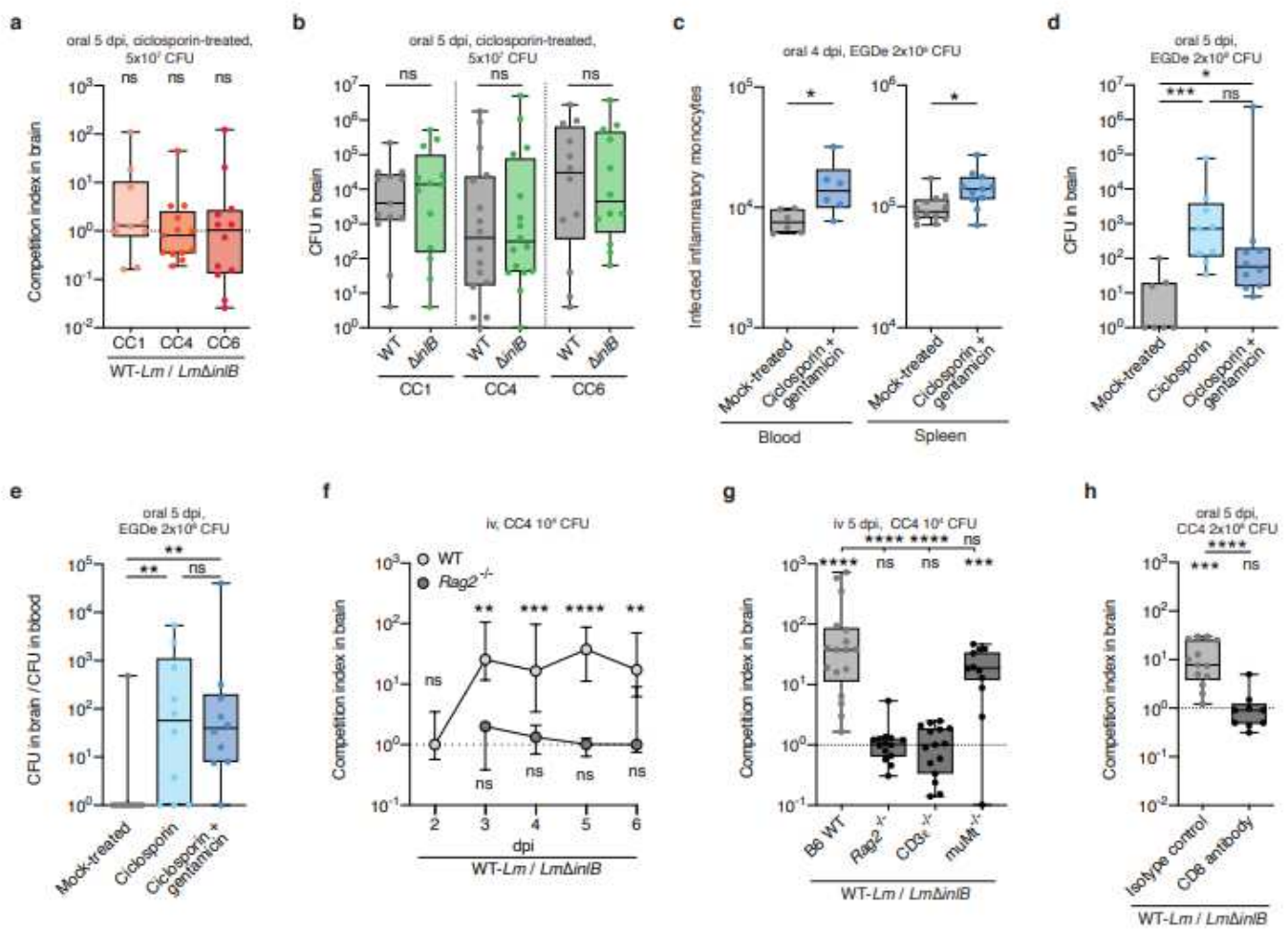

i

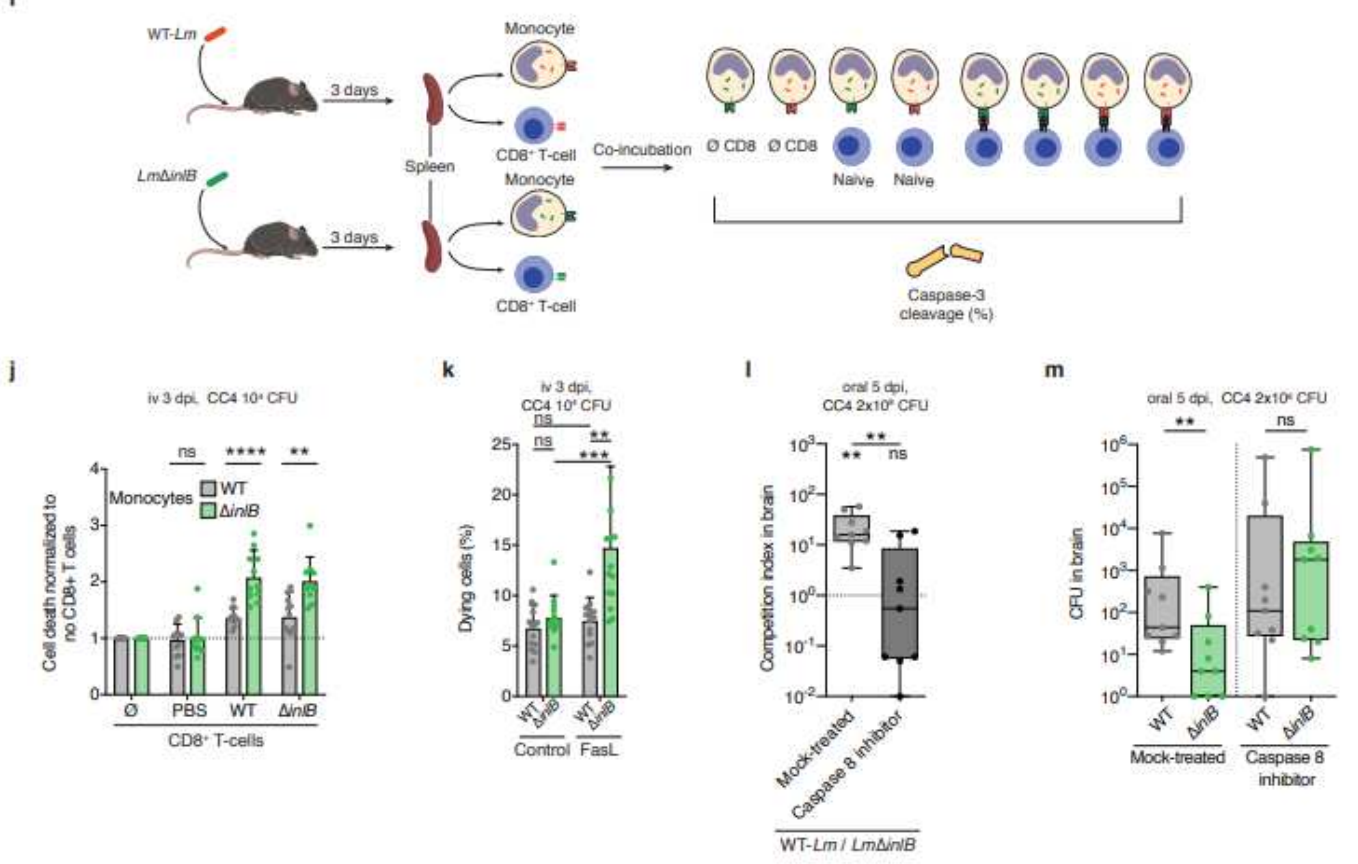

Figure 3

InIB blocks CD8+ T-cells-mediated monocyte cell death. 
a

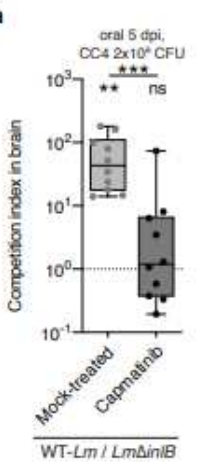

f
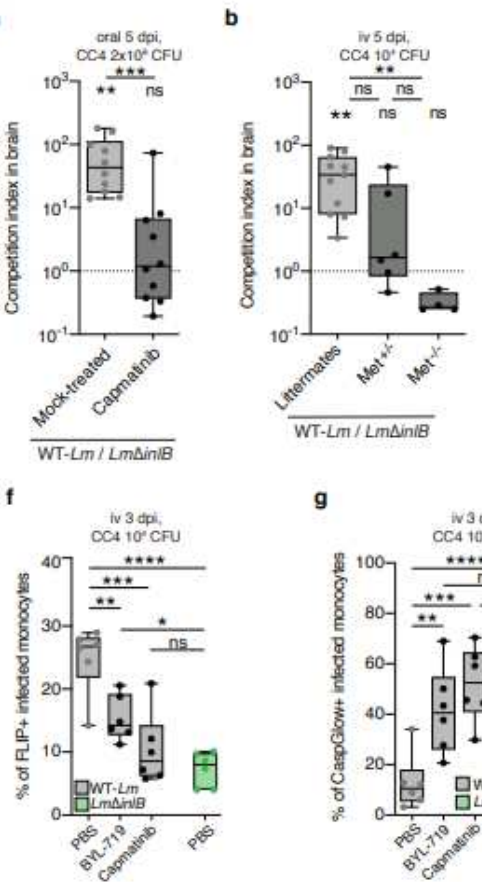

g

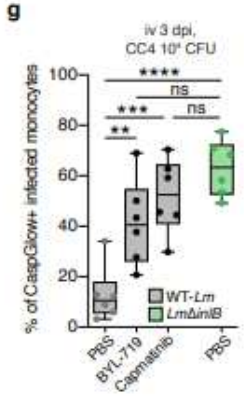

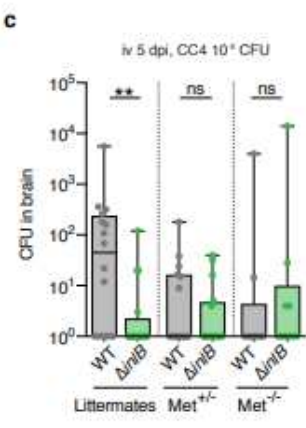

d

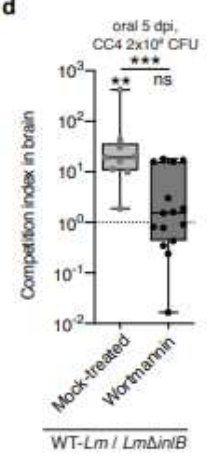

h

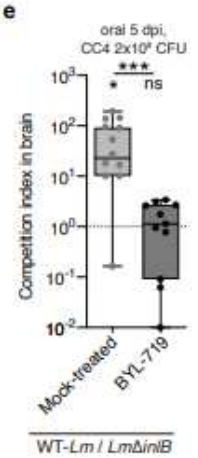

i

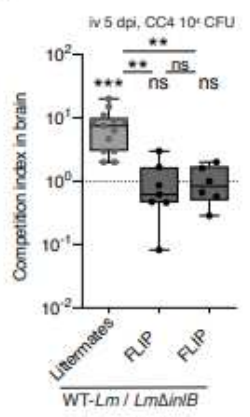

j

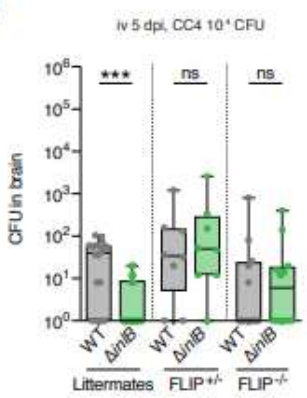

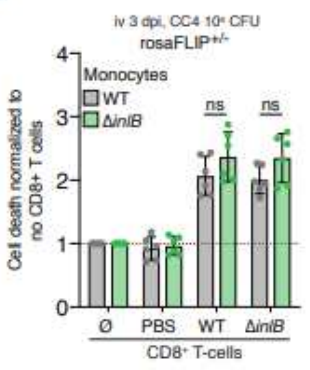

k

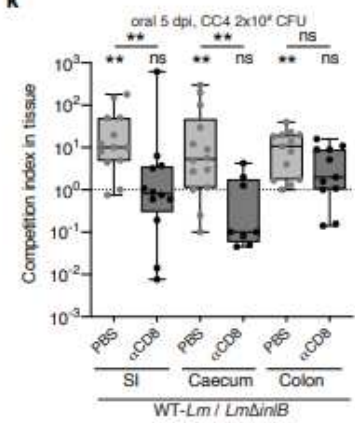

I

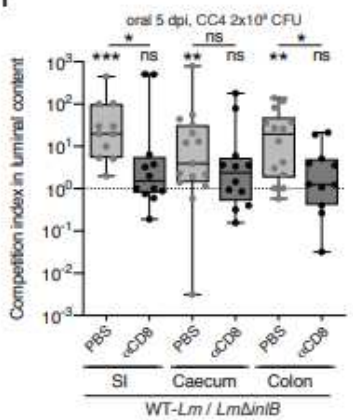

m

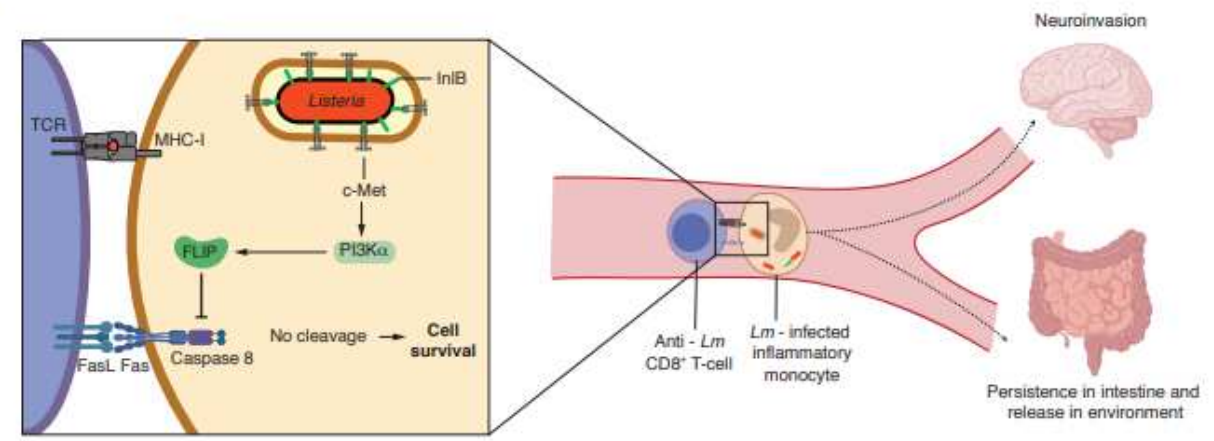

Figure 4

Inhibition of CD8+ T cells-mediated cell death by InIB is dependent on the c-Met-PI3Ka-FLIP pathway and is involved in Lm intestinal colonization and fecal carriage.

\section{Supplementary Files}


This is a list of supplementary files associated with this preprint. Click to download.

- ExtendedDataMovie1.mp4

- MaudetExtendedData.pdf

- MaudetMethodsTables.pdf 